\title{
Antigenic escape selects for the evolution of higher pathogen transmission and virulence
}

\begin{abstract}
Akira Sasaki ${ }^{1,2}$, Sébastien Lion $\mathbb{1}^{3}$ and Mike Boots $\mathbb{1}{ }^{4,5} \bowtie$
Despite the propensity for complex and non-equilibrium dynamics in nature, eco-evolutionary analytical theory typically assumes that populations are at equilibria. In particular, pathogens often show antigenic escape from host immune defences, leading to repeated epidemics, fluctuating selection and diversification, but we do not understand how this impacts the evolution of virulence. We model the impact of antigenic drift and escape on the evolution of virulence in a generalized pathogen and apply a recently introduced oligomorphic methodology that captures the dynamics of the mean and variance of traits, to show analytically that these non-equilibrium dynamics select for the long-term persistence of more acute pathogens with higher virulence. Our analysis predicts both the timings and outcomes of antigenic shifts leading to repeated epidemics and predicts the increase in variation in both antigenicity and virulence before antigenic escape. There is considerable variation in the degree of antigenic escape that occurs across pathogens and our results may help to explain the difference in virulence between related pathogens including, potentially, human influenzas. Furthermore, it follows that these pathogens will have a lower $R_{0}$, with clear implications for epidemic behaviour, endemic behaviour and control. More generally, our results show the importance of examining the evolutionary consequences of non-equilibrium dynamics.
\end{abstract}

nfectious diseases remain a major problem for human health and agriculture ${ }^{1-4}$ and are increasingly recognized as important in ecosystems and conservation ${ }^{5}$. This has led to the development of extensive theoretical literature on the epidemiology, ecology and evolution of host-pathogen interactions $\mathrm{s}^{6-9}$. Understanding the drivers of the evolution of virulence, typically defined in the evolutionary literature as the increased death rate of individuals due to infection, is a key motivator of this theoretical work ${ }^{7,9-13}$. Generally, models assume that a higher transmission rate trades off against the intrinsic cost of reducing the infectious period due to higher death rates (virulence), and classically predict the evolution of virulence that maximizes the parasite epidemiological $R_{0}^{7,9-13}$. In fact, this result only holds in models where ecological feedbacks take a constrained form, such that even relatively simple processes such as density-dependent mortality, multiple infections and spatial structure may lead to diversification or different optima ${ }^{9,11,12,14}$. Moreover, this classic evolutionary theory examines the long-term equilibrium evolutionary outcome in the context of stable endemic diseases; however, in nature, infectious diseases often exhibit complex dynamics, with potentially important impacts on pathogen fitness $^{15-18}$

Antibody-mediated immunity is a critical factor driving the dynamics of important infectious diseases such as seasonal influenza, leading to selection for novel variants that can escape immunity to the current predominant variant ${ }^{19-21}$. Such antigenic escape typically causes the optimal variant of the parasite to change through time as it moves through antigenic space. Moreover, partial cross immunity between the different parasite variants may lead to recurrent epidemics, fluctuations in parasite variants and potentially, variant coexistence ${ }^{22-25}$. Previous theory has shown that the evolution of immune escape can lead to dramatic disease outbreaks $^{23-25}$, but the implications of these epidemiological dynamics for the evolution of disease virulence are unknown. This question is challenging, in part because much of the theoretical framework used to study virulence evolution typically considers diseases that are at an endemic equilibrium ${ }^{7,9-13}$. As such, we currently lack a broad theoretical understanding of the evolution of virulence in the presence of antigenic escape, despite its importance as an epidemic process and the probable implications of its inherently dynamical epidemic nature.

Here we examine the impact of antigenic escape on the evolution of infectious disease in the context of the well-studied transmission-virulence trade-off $f^{9,26}$. We first analytically examine the case without cross immunity and then apply a recently introduced 'oligomorphic' analysis that combines quantitative genetic and game theoretical approaches ${ }^{27}$ to examine the impact of antigenic jumps and epidemic outbreaks due to cross immunity. Specifically, oligomorphic analysis explicitly models not only changes in the mean trait but also changes in the variance of the trait. This variance is critical to the evolutionary outcome under non-equilibrium dynamics and the approach allows us to model the evolutionary dynamics of populations with multiple peaks in the character distribution. This analysis can be applied across a range of ecological and evolutionary timescales and allows us to examine evolutionary outcomes under non-equilibrium conditions. Our key result is that antigenic escape selects for higher transmission and virulence due to the repeated epidemics caused by immune escape, leading to the long-term persistence of acute pathogens. Indeed, antigenic escape has the potential to select for infectious diseases with substantially higher virulence than that predicted by the maximization of $R_{0}$ in classic disease models.

\section{Results}

To tractably model antigenic escape with multiple variants and cross immunity, we follow the simplifying approaches of Gog and Grenfell ${ }^{25}$. Effectively, the role of cross immunity is to generate protection against

'Department of Evolutionary Studies of Biosystems, The Graduate University of Advanced Studies, SOKENDAI, Hayama, Kanagawa, Japan. ${ }^{2}$ Evolution and Ecology Program, International Institute for Applied Systems Analysis, Laxenburg, Austria. ${ }^{3}$ CEFE, CNRS, Univ Montpellier, EPHE, IRD, Montpellier, France. ${ }^{4}$ Integrative Biology, University of California, Berkeley, CA, USA. ${ }^{5}$ Biosciences, University of Exeter Penryn Campus, Cornwall, UK. $凶_{\mathrm{e}}$-mail: mboots@ berkeley.edu 


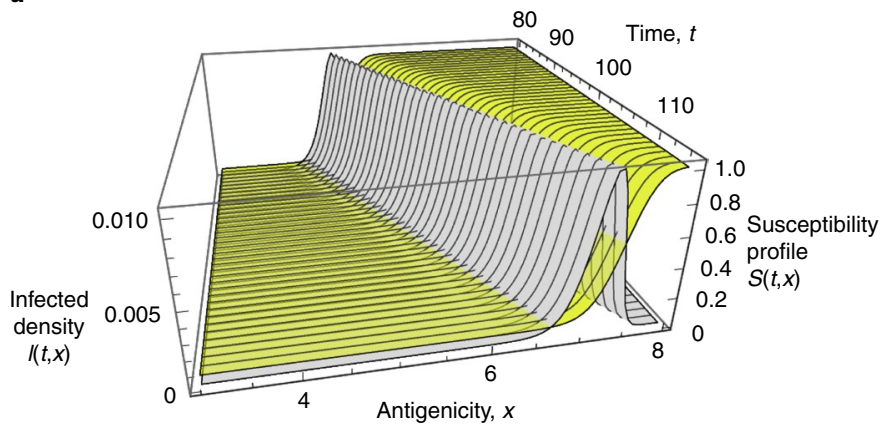

b

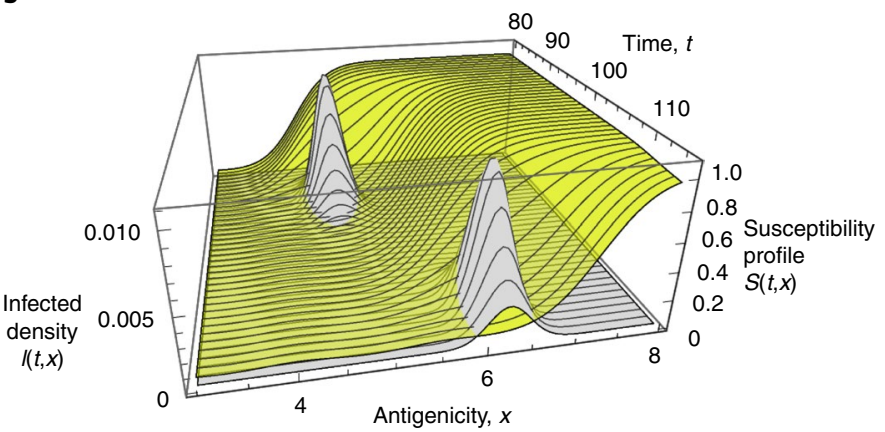

Fig. 1 | Continuous antigenic drift (a) and periodic antigenic shifts (b) of the model. The grey coloured surface denotes the infected density $I(t, x)$ varying in time $t$ and antigenicty $x$, and the yellow coloured surface denotes the density of hosts $S(t, x)$ that are susceptible to antigenicity variant $x$ of pathogen at time $t$. The cross immunity $\sigma(x)=\exp \left(-x^{2} / 2 \omega^{2}\right)$ has a width of $\omega=0.2$ in a and $\omega=0.6$ in $\mathbf{b}$. Other parameters are $\beta=2, \alpha=0.1, \gamma=0.5$, and $D=0.001$.

becoming infectious with variants not yet encountered. In particular, we assume that cross immunity reduces the transmissibility of, rather than the susceptibility to, future variants. Furthermore, we assume that there is polarizing immunity, such that cross immunity results in a proportion of individuals being completely immune. These assumptions allow tractability and have been shown not to impact the predictions of the mode ${ }^{25}$. Specifically, we consider a population of pathogens structured by a one-dimensional antigenic trait $x$, so that $I(t, x)$ is the density of hosts infected with antigenicity variant $x$ at time $t$. Following Gog and Grenfell ${ }^{25}$, we assume that an individual is either perfectly susceptible or perfectly immune to a variant. A variant of pathogen can infect any host, but will be infectious only when the host is susceptible to that variant. When a variant $y$ of pathogen infects a host that is susceptible to a variant $x$, the host may become (perfectly) immune to the variant $x$ with probability $\sigma(x-y)$. This is the partial cross immunity function between variants $x$ and $y$, which takes a value between 0 and 1 and is a decreasing function of antigenic distance $|x-y|$ between variants $x$ and $y$. The density of hosts susceptible to antigenicity variant $x$ at time $t$ is noted as $S(t, x)$.

Assuming that all pathogen variants have the same transmission rate $\beta$ and virulence $\alpha$, we can describe the dynamics with the following structured 'susceptible-infected-recovered' model:

$$
\begin{gathered}
\frac{\partial S(t, x)}{\partial t}=-\beta S(t, x) \int_{-\infty}^{\infty} \sigma(x-y) I(t, y) d y, \\
\frac{\partial I(t, x)}{\partial t}=[\beta S(t, x)-(\gamma+\alpha)] I(t, x)+D \frac{\partial^{2} I(t, x)}{\partial x^{2}},
\end{gathered}
$$

where $\gamma$ is the recovery rate and $D=\mu \sigma_{\mathrm{m}}^{2} / 2$ is the diffusion constant due to random mutation in the continuous antigenic space, which is defined by one half of the mutation variance $\mu \sigma_{\mathrm{m}}^{2}$, where $\mu$ is the mutation rate and $\sigma_{\mathrm{m}}^{2}$ is the variance in the difference between parental and mutant traits ${ }^{28,29}$. The dynamics for the density of recovered hosts is omitted from equation (1) as it does not affect the dynamics of the densities of susceptible and infected hosts.

Invasion of a single pathogen. In our first scenario, we start with a population where all hosts are susceptible to any variant $(S(0, x)=1)$ and a small number of hosts infected by pathogen variant with antigenicity trait $x=0$ is initially introduced. The system then exhibits travelling wave dynamics in antigenicity space ${ }^{30}$. At the front of the travelling wave, $I(t, x)$ is sufficiently small and $S(t, x)$ is sufficiently close to 1. Equation (1a) can then be linearized as

$$
\frac{\partial I(t, x)}{\partial t}=r I(t, x)+D \frac{\partial^{2} I(t, x)}{\partial x^{2}},
$$

where $r=\beta-(\gamma+\alpha)$ is the rate of increase of an antigenicity variant before it spreads in the population and causes the build-up of herd immunity. The system (equation 1) asymptotically approaches travelling waves of both pathogen antigenicity distributions $I(t, x)$, which have an isolated peak around the current antigenicity and host susceptibility profile $S(t, x)$, which smoothly steps down towards a low level after the pathogen antigenicity distribution passes through, with a common constant wave $\operatorname{speed~}^{31}$ (Fig. 1a)

$$
v=2 \sqrt{r D}=2 \sqrt{(\beta-(\gamma+\alpha)) D} .
$$

As the width of the partial cross-immunity function $\sigma(x-y)$ increases, the travelling wave with static shapes described above is destabilized (Extended Data Fig. 1), and the system shows intermittent outbreaks that occur periodically both in time and in antigenicity space ${ }^{25,31}$ (Fig. 1b). However, the wave speed is unchanged from equation (3), as the linearized system (equation 2) towards the frontal end remains the same irrespective of the stability of the wave profile that lags behind (Extended Data Fig. 1).

Evolution of antigenic escape with cross immunity. To predict how cross immunity affects the evolution of antigenic escape, we use an oligomorphic dynamics analysis ${ }^{27}$. In this analysis, we consider a population composed of different antigenicity 'morphs' that can be seen as quasi-species. Specifically, we use the term 'morph' to describe the phenotypic trait mean and the continuous variance around this mean. The analysis in the methods allows us to track the dynamics of morph frequencies, $p_{i}$, and mean trait values, $\bar{x}_{i}$, as:

$$
\begin{gathered}
\frac{d p_{i}}{d t}=\beta\left(\bar{s}_{i}-\bar{s}\right) p_{i}, \\
\frac{d \bar{x}_{i}}{d t}=V_{i} \beta s \prime\left(\bar{x}_{i}\right),
\end{gathered}
$$

where $s(x)$ is the susceptibility profile of the population, which depends on the cross-immunity function $\sigma, \bar{s}_{i}$ is the mean susceptibility perceived by viral morph $i$, and $\bar{s}$ the mean susceptibility averaged over the different viral morphs. Note that, in general, $s(x)$, $\bar{s}_{i}$ and $\bar{s}$ will be functions of time, as the susceptibility profile is moulded by the epidemiological dynamics of $S(t, x)$ and $I(t, x)$.

Equation (4a) reveals that, as intuitively expected, morph $i$ will increase in frequency if the susceptibility of the host population to this variant is higher on average. Equation (4b) shows that the increase in the mean antigenicity trait of morph $i$ depends on: (1) the variance of the morph distribution, $V_{i}$; (2) the transmission rate; and (3) the slope of the susceptibility profile close to the morph mean $\bar{x}_{i}$. Together with an equation for the dynamics of variance under mutation and selection (see Methods), equations (4a) and 

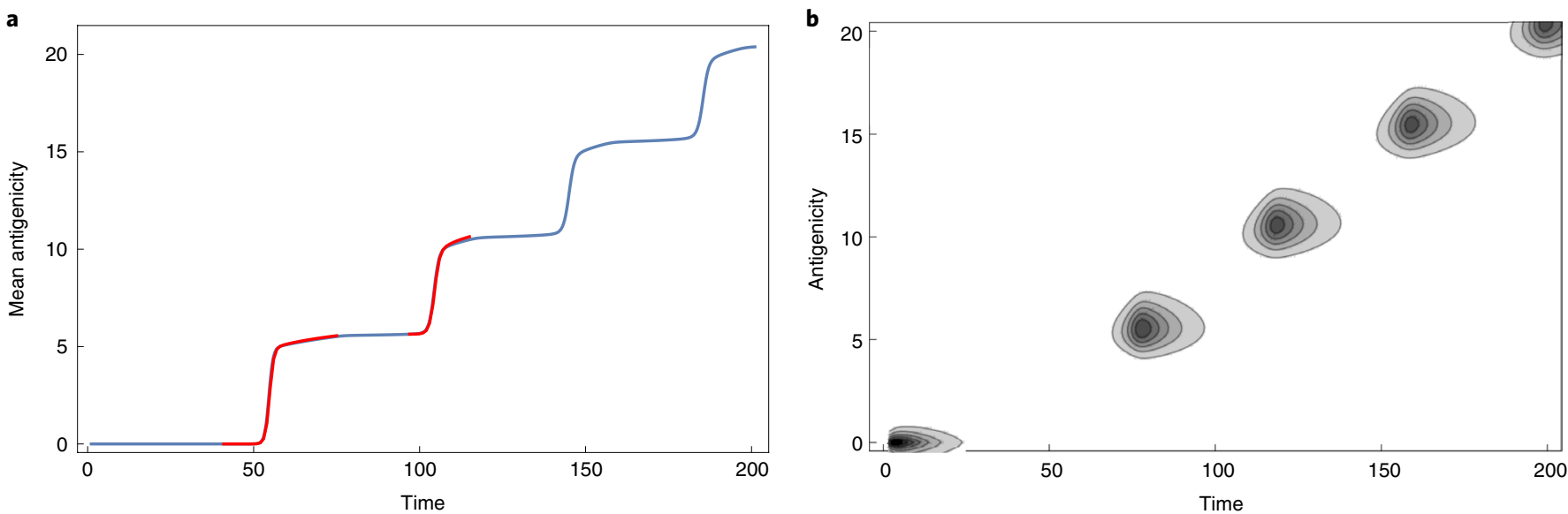

Fig. 2 | Oligomorphic dynamics prediction of the emergence of antigenicity shift. a, Oligomorphic prediction for the change in the mean antigenicity after the primary outbreak at $x=0$ and the change after the second outbreak starting around $x=5.5$ (red curves), compared with that obtained by numerical simulations (blue curves). b. Heat map representation of the time change of the antigenic drift model (equation 1). Parameters: $\beta=2, \gamma+\alpha=0.6$, $\sigma(x)=\exp \left(-x^{2} / 2 \omega^{2}\right)$ with $\omega=2, D=0.001$. Initially, all hosts are equally susceptible, with $S(0, x)=1$. The primary pathogen variant is introduced at $x=$ 0 , with infected density 0.001 .

(4b) allow us to quantitatively predict the change in antigenicity after a primary outbreak, as shown in Fig. 2.

For instance, after a primary outbreak caused by a variant with antigenicity $\bar{x}_{0}=0$ at $t=0$, the susceptibility profile is approximately constant and given by $s(x)=\left(1-\psi_{0}\right)^{\sigma(x)}$, where $\psi_{0}$ is the final size of the epidemic of the primary outbreak at antigenicity $x$ $=0$ (see Methods). Thus, for a decreasing cross-immunity function, $\sigma(x)$, the slope of the susceptibility profile is positive, which selects for increased values of the mean antigenicity trait $\bar{x}_{1}$ of a second emerging morph (see Methods). As the process repeats itself, this leads to successive jumps in antigenic space. In addition, a more peaked cross-immunity function, $\sigma$, yields larger slopes to the susceptibility profile and thus selects for higher values of the antigenicity trait.

Long-term joint evolution of antigenicity, transmission and virulence. We now extend our analysis to account for mutations affecting pathogen life-history traits such as transmission and virulence. To simplify, we use the classical assumption of a transmission-virulence trade-off ${ }^{7,-13}$ and consider that a pathogen morph, $i$, has frequency, $p_{i}$, mean antigenicity trait, $\bar{x}_{i}$, and mean virulence $\bar{\alpha}_{i}$. In the methods, we show that the morph's mean traits change as

$$
\frac{d}{d t}\left(\begin{array}{c}
\bar{x}_{i} \\
\bar{\alpha}_{i}
\end{array}\right)=G_{i}\left(\begin{array}{c}
\beta\left(\bar{\alpha}_{i}\right) s \prime\left(\bar{x}_{i}\right) \\
\beta \prime\left(\bar{\alpha}_{i}\right) s\left(\bar{x}_{i}\right)-1
\end{array}\right)
$$

where $G_{i}$ is the genetic (co)variance matrix, and the vector on the right-hand side is the selection gradient. Note that, while the selection gradient on antigenicity depends on the slope of the antigenicity profile at the morph mean, the selection gradient on virulence depends on the slope of the transmission-virulence trade-off at the morph mean, weighted by the susceptibility profile at the morph mean.

Assuming we can neglect the build-up of correlations between antigenicity and virulence due to mutation and selection, the genetic (co)variance matrix is diagonal with elements $V_{i}^{x}$ and $V_{i}^{\alpha}$. Then, as shown previously, antigenicity increases if the slope of the susceptibility profile is locally positive, while mean virulence increases as long as $\beta \prime\left(\bar{\alpha}_{i}\right)>1 / s\left(\bar{x}_{i}\right)$. For a fixed antigenicity trait, $x=x^{*}$, the susceptibility profile converges towards $s\left(x^{*}\right)=(\gamma+\alpha) / \beta$ and the evolutionary endpoint satisfies

$$
\beta \prime(\alpha)=\frac{\beta(\alpha)}{\gamma+\alpha},
$$

which corresponds to the classical result of $R_{0}$ maximization for the unstructured susceptible infected (SI) model ${ }^{14,26}$. However, when antigenicity can evolve, selection will also lead to the build-up of a positive covariance $C$ between antigenicity and virulence, resulting in a synergistic effect (Methods). As the antigenicity trait increases, the evolutionary trajectory of virulence converges to the solution of

$$
\beta^{\prime}(\alpha)=1,
$$

which corresponds to maximizing the rate of increase of pathogen $r(\alpha)=\beta(\alpha)-(\gamma+\alpha)$ in a fully susceptible population. This is equivalent to maximizing the wave speed $v(\alpha)=2 \sqrt{r(\alpha) D}$, as shown in Methods. Figure 3a shows that, in the absence of cross immunity, the evolutionarily stable (ES) virulence is well predicted by $r$ maximization. With cross immunity (Fig. 3b), virulence evolution is characterized by jumps that reflect the sudden shifts in antigenicity due to cross immunity.

As such antigenic escape selects for higher transmission and virulence and more acute infectious diseases. This has parallels with the results that show that there is a transient increase in virulence at the start of an epidemic with $r$ rather than $R_{0}$ being maximized $^{15,16,18,32}$, but here we predict the long-term persistence of highly transmissible and virulent disease variants due to antigenic escape.

Although we have so far assumed a never-ending antigenic escape process, it is easy to extend our analysis to consider that antigenic escape is constrained by pleiotropic effects. Then, once the antigenicity trait has stabilized, the ES virulence would satisfy

$$
\beta \prime(\alpha)=\frac{1-\rho \beta(\alpha) s \prime(x)}{s(x)},
$$

where $\rho=C / V_{\alpha}$ measures the correlation between antigenicity and virulence. Thus, the slope to the transmission-virulence trade-off at the evolutionarily stable strategy (ESS) now takes an intermediate value between $\beta /(\gamma+\alpha)$ and 1 , as shown in Fig. 4 .

Short-term joint evolution of antigenicity and virulence. Although our analysis allows us to understand the long-term evolution of pathogen traits, it can also be used to accurately predict 
a

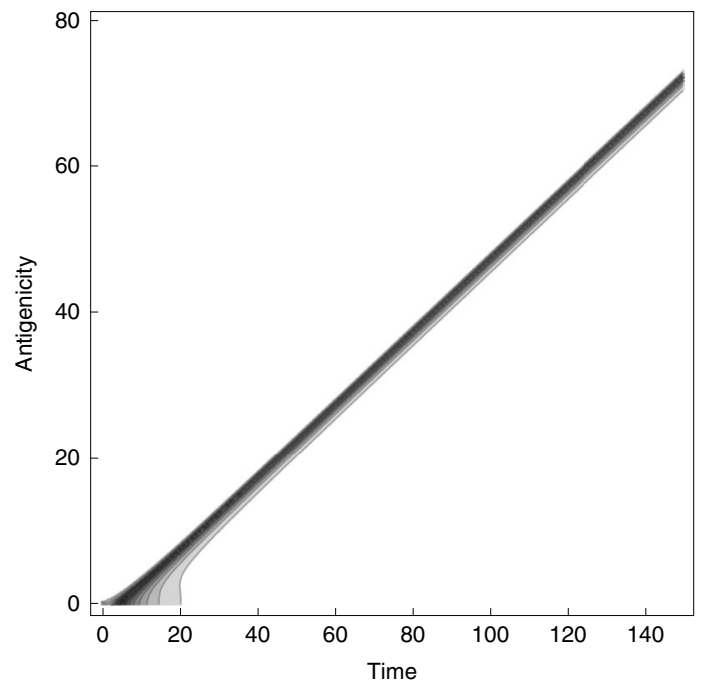

b

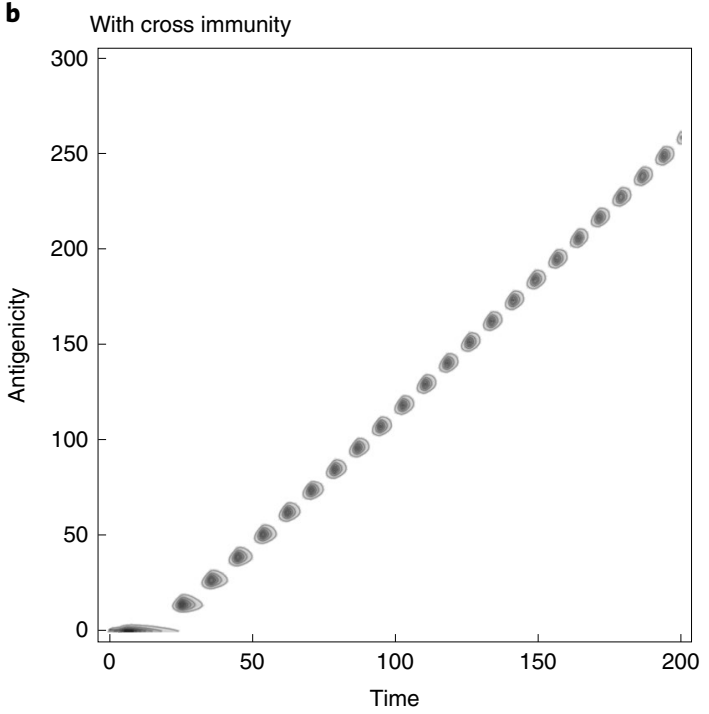

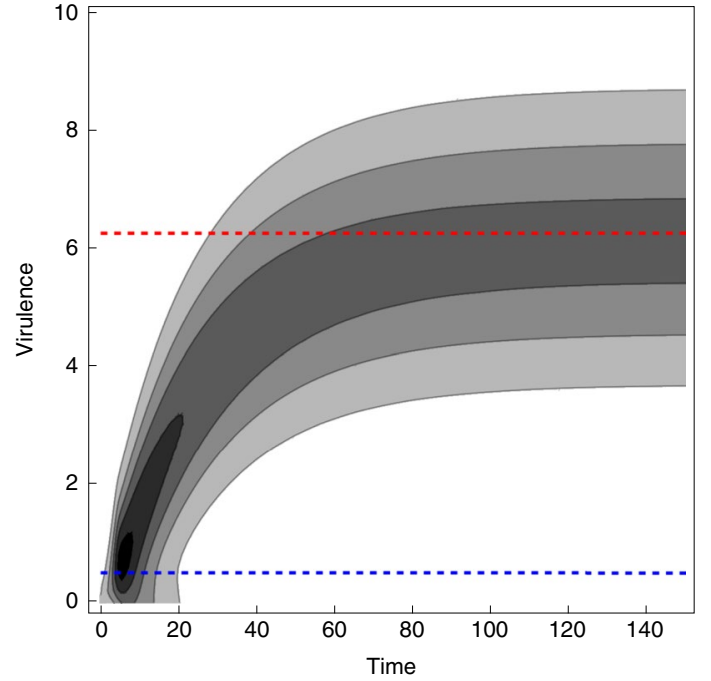

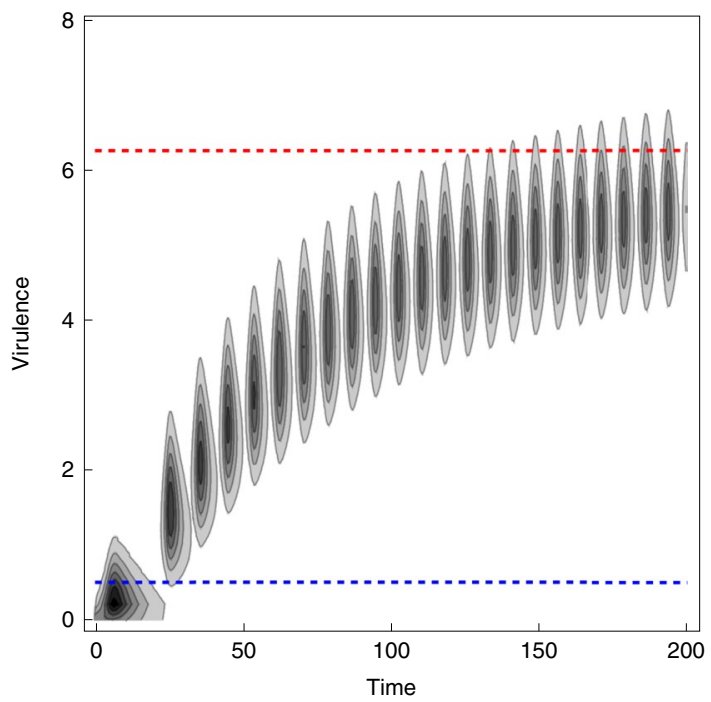

Fig. 3 | Marginal distribution of antigenicity (left) and virulence (right) in the absence or presence of cross immunity. a, No cross immunity is assumed so that each antigenicity genotype causes specific herd immunity: $\sigma(x-y)=\delta(x-y)$, where $\delta(\cdot)$ is Dirac's delta function. There are 1,600 antigenic variations having equally divided antigenicity between 0 and 80 . b. We assume a Gaussian cross-immunity kernel, $\sigma(x-y)=\exp \left(-(x-y)^{2} / 2 \omega^{2}\right)$, with width $\omega=5$. There are 300 antigenic variations having equally divided antigenicity between 0 and 300 . In both $\mathbf{a}$ and $\mathbf{b}$, there are 100 viral virulence traits, each having virulence equally divided between 0 and 20, and diffusion constants due to mutations are $D_{x}=0.01$ (for antigenicity) and $D_{\alpha}=0.01$ (for virulence). The blue dashed lines show the ES virulence predicted from maximizing $R_{0}$, as expected in the absence of antigenic escape, while the red dashed lines show the predicted ES virulence that maximizes $r(\alpha)=\beta(\alpha)-(\gamma+\alpha)$, as predicted from our analysis. Other parameters: $\gamma=0.5$, and $\beta(\alpha)=5 \sqrt{\alpha}$.

the short-term dynamics of antigenicity and virulence. We now consider that a primary outbreak has moulded a susceptibility profile $s(x)$ that we assume constant. Although this assumption will cause deviations from the true susceptibility profile, it allows us to decouple our evolutionary oligomorphic dynamics from the epidemiological dynamics. Figure 5 shows that the approximation accurately predicts the jump in antigenic space and joint increase in virulence during the secondary outbreak. The accuracy of the prediction depends on the time at which we seed the oligomorphic dynamical system, as detailed in the methods, but remains high for a broad range of values of this initial time. Hence, our analysis can be used to successfully predict the trait dynamics after the emergence of a new antigenic variant. Simulations show that this result is not dependent on the assumption of a one-dimensional antigenic space (Supplementary Information).

\section{Discussion}

We have shown how antigenic escape selects for more acute infectious diseases with higher transmission rates that cause increased mortality (virulence) in infected hosts. This result is important given the number of major infectious diseases, such as seasonal influenza, that have epidemiology driven by antigenic escape. Until recently, the evolution of virulence literature has mostly focused on equilibrium solutions that, in simple models, lead to the classic idea that pathogens evolve to maximize their basic reproductive number $R_{0}^{7,9-13}$. Our results show that the process of antigenic escape leading to the continual replacement of variants ${ }^{22-25}$, creates a dynamical invasion process that in itself selects for more acute, fast transmitting, highly virulent variants that do not maximize $R_{0}$. This has parallels with the finding that more acute variants are selected transiently at the start of epidemics ${ }^{23-25}$, but critically, in 


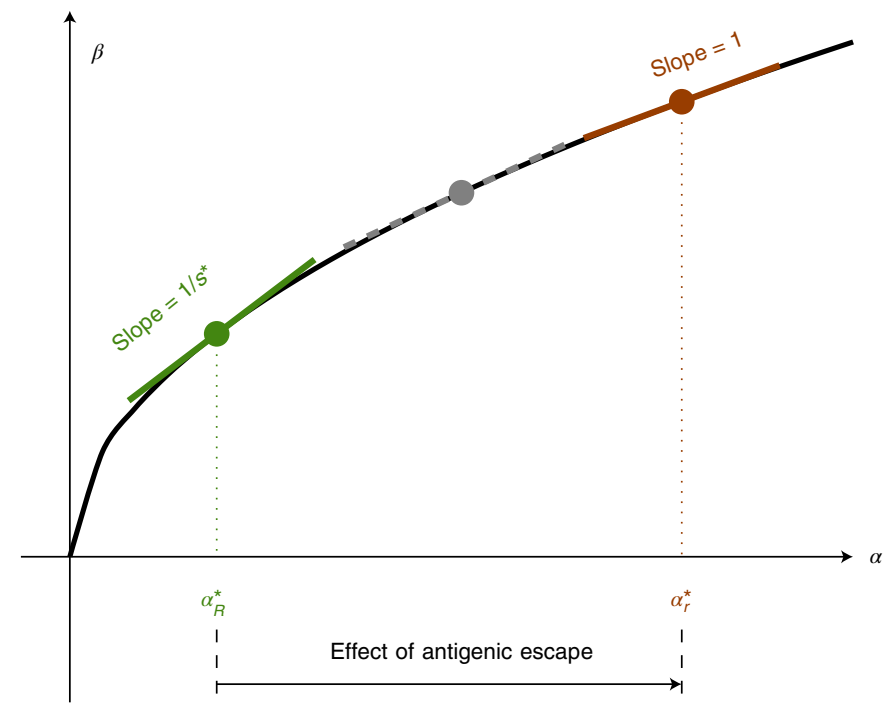

Fig. 4 | Graphical representation of the predicted ES virulence with or without antigenic escape under the assumption of a transmission-virulence trade-off. In the absence of antigenic escape, the ES virulence, $\alpha_{R^{\prime}}^{*}$ can be predicted from the maximization of the pathogen's epidemiological basic reproduction ratio, $R_{0}=\beta /(\gamma+\alpha)$, or equivalently by the minimization of the total density of susceptible hosts since, at equilibrium, $s^{*}=1 / R_{0}$. The slope of the transmission-virulence trade-off at the ESS is then $1 / s^{*}=R_{0}$. With antigenic escape, the ES virulence, $\alpha_{r}^{*}$, can be predicted from the maximization of the pathogen's growth rate in a fully susceptible population, $r_{0}=\beta(\alpha)-(\gamma+\alpha)$. The slope of the transmission--virulence trade-off at the ESS is then 1. This holds true in the limit of a large antigenicity trait, but intermediate values of ES virulence, corresponding to intermediate slopes, can also be selected for if other processes constrain the evolution of the antigenicity trait, as explained in the main text.

our case the result is not a short-term transient outcome. Rather, the eco-evolutionary process leads to the long-term persistence of more acute variants. As such, antigenic escape may be an important driver of high virulence in infectious disease.

In the simpler case where there is no cross immunity, there is a travelling wave of new variants invading due to antigenic escape. In this case, we can use established methods to gain analytical results that not only predict the speed of change of the variants, but also the evolutionarily stable virulence. With our model's assumptions, without antigenic escape, we would get the classic result of the maximization of the reproductive number $R_{0}^{7,9-13}$, but once there is antigenic escape, we show analytically that the intrinsic growth rate of the infectious disease $r$ is maximized. Maximizing the intrinsic growth rate leads to selection for higher transmission and, in turn, higher virulence. Effectively, this is the equivalent 'live fast, die young' strategy of an infectious disease. The outcome is due to the dynamical replacement of variants, with new variants invading the population continually, leading to a continual selection for the variants that invade better ${ }^{23-25}$. As such, we predict that any degree of antigenic escape will, in general, select for more acute faster-transmitting variants with higher virulence in the presence of a transmission-virulence trade-off. Although such a trade-off is a classical assumption in evolutionary epidemiology, it would be interesting to examine the impact of antigenic escape under different assumptions.

Partial cross immunity leads to a series of jumps in antigenic space that are characteristic of the epidemiology of a number of diseases and, in particular, of the well-known dynamics of influenza $\mathrm{A}$ in humans ${ }^{22-25}$. Here a cloud of variants remains in antigenic space until there is a jump that, on average, overcomes the cross immunity and leads to the invasion of a new set of variants that are distant enough to escape the immunity of the resident variants ${ }^{22-25}$. To examine the evolutionary outcome in this scenario, we applied a novel oligomorphic analysis ${ }^{27}$ and again we find that antigenic escape selects for higher virulence towards the maximization of the intrinsic growth rate $r$. Both our analysis and simulations show that in the long term, virulence increases until it reaches a new optimum, potentially of an order of magnitude higher than would be expected by the classic prediction of maximizing $R_{0}$. Therefore, antigenic escape, whether it is through a continuous wave of antigenic drift or through large jumps, selects for higher virulence. We therefore expect this result to apply across the wide range of 'jumpiness' that we see across different viruses between these two extremes of continuous drift and punctuated jumps. We show that virulence increases after each antigenic jump, falling slightly at the next jump before increasing again until it reaches this new equilibrium. It is also important to note that the diversity within the morph increases in both antigenicity and virulence as we move towards the next epidemic, reaching a maximum just at the point when the jump occurs. This increase in diversity could, in principle, be used as a predictor of the next jump in antigenic space.

Clearly, the virulence of any particular infectious disease depends on multiple factors, including both host and parasite traits, and critically, the relationship between transmission and virulence. This makes comparisons of virulence across different infectious diseases problematic since the specific trade-off relationship between transmission and virulence is often unknown. However, our model shows that antigenic escape will, all things being equal, be a driver of higher virulence favouring more acute variants. It is also important to note that since antigenic escape is a very general mechanism that selects for higher virulence, it follows that we may see high virulence in parasites even when the costs in terms of reduced infectious period are substantial. Among the influenzas, although there is paucity of data, influenza $\mathrm{C}$ does not show obvious antigenic escape and is typically much less virulent than the other influenzas $^{33}$. Furthermore, influenza A/H3 tends to show much more antigenic escape than influenza $B$ and influenza $A / H 1$, and again in line with our predictions, influenza $\mathrm{A} / \mathrm{H} 3 \mathrm{~N} 2$ is typically the more virulent type $\mathrm{e}^{34,35}$. It is important to note that these differences can be ascribed to multiple factors, including circumvention of vaccination, and that cross immunity may itself directly impact disease severity. Furthermore, the higher virulence of influenza A is often posited to be due to a more recent zoonotic emergence ${ }^{36}$. Moreover, direct comparisons of the rates of antigenic escape between influenza $\mathrm{A} / \mathrm{H} 1$ and influenza $\mathrm{B}$ are difficult, and clearly, there are also highly virulent pathogens that do not show antigenic escape, such as measles. Therefore, a formal comparative analysis is confounded by multiple factors. Nevertheless, our model suggests that differences in the rates of antigenic escape of the different influenzas may impact their virulence and the evidence from influenza is at least consistent with the predictions of the model.

An important implication of our work is that antigenic escape selects for variants with a higher virulence than the value that maximizes $R_{0}$ and therefore leads to the evolution of infectious diseases with lower $R_{0}$. From this point of view, it may be naively concluded that diseases with antigenic escape may be easier to eliminate and control with vaccination. Of course, in practice the opposite is often true since producing an effective vaccine is much more problematic when there is antigenic escape ${ }^{37,38}$. On the other hand, with lower $R_{0}$, epidemics will tend to be less explosive than they otherwise would be, having a lower peak but lasting longer, with evolution here effectively 'flattening the curve'. Infectious diseases that show punctuated antigenic escape are characterized by repeated epidemics, but our work suggests that due to the selection for a lower $R_{0}$, the eco-evolutionary feedback would have substantially impacted the pattern of these epidemics. More generally our results highlight 
a

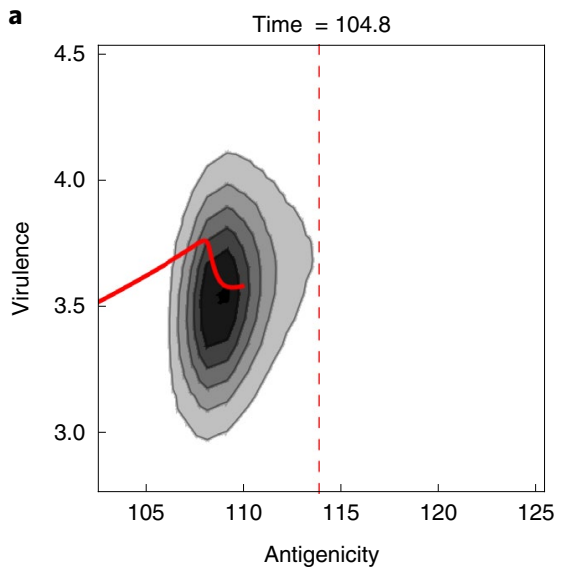

b

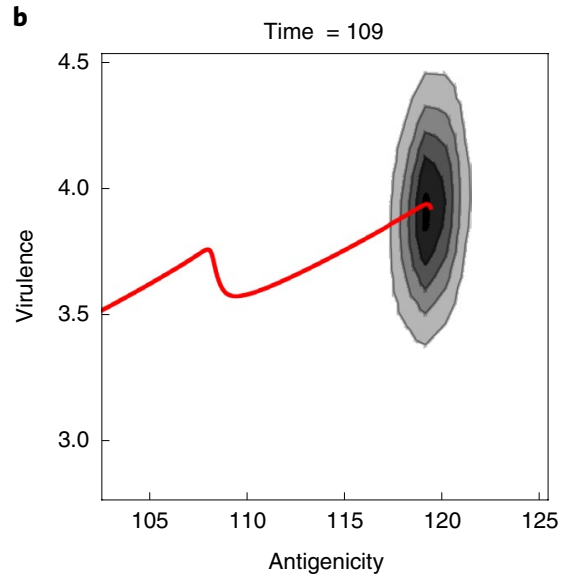

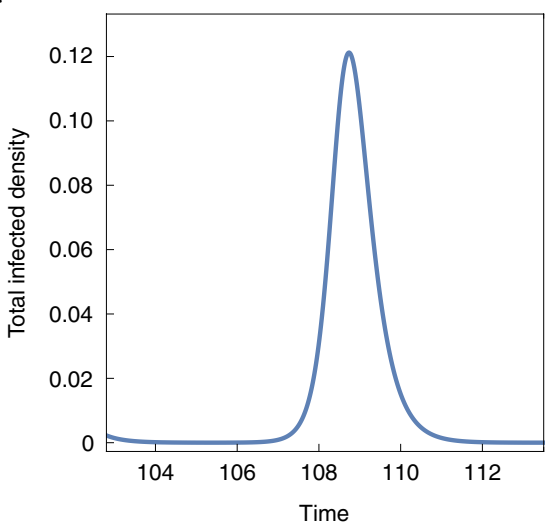

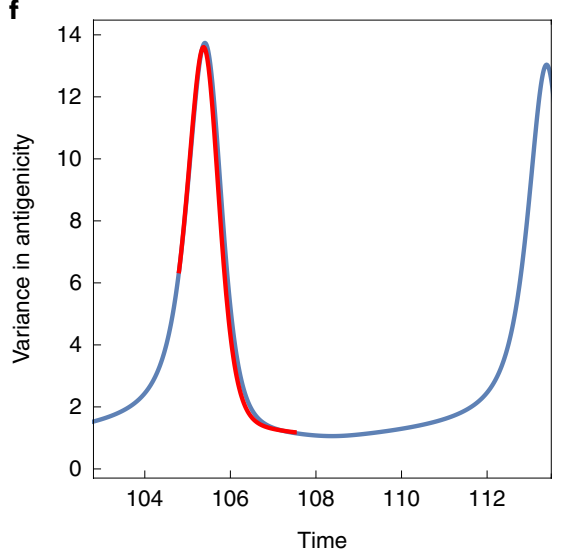

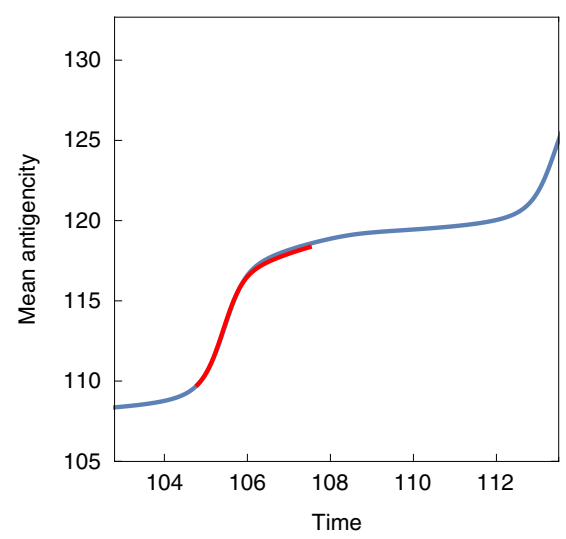

g

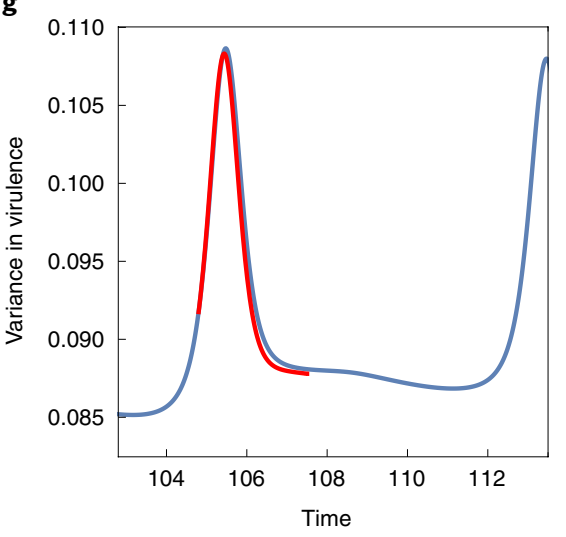

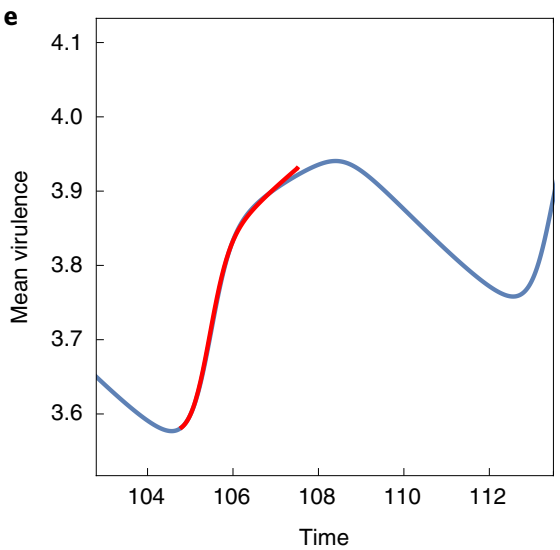

h

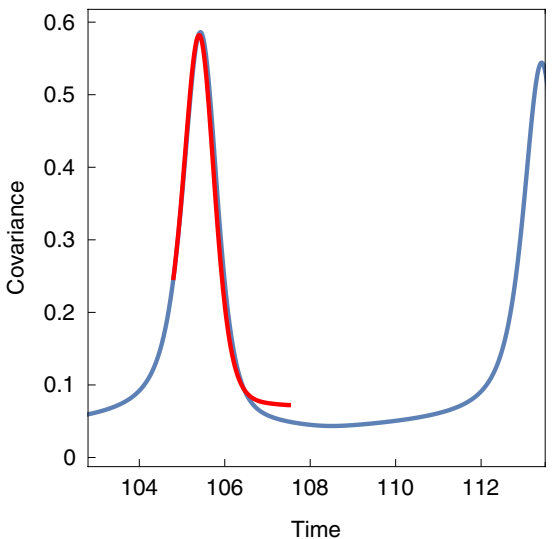

Fig. 5 | Oligomorphic dynamics predictions compared to the simulation results of the emergence of the next variant during antigenicity-virulence coevolution. $\mathbf{a}, \mathbf{b}$, Simulation results showing snapshots of contour plots for the joint trait distribution observed near the starting (a) and finishing (b) time of emergence. The overlaid curves are the trajectory of mean traits $(\bar{x}, \bar{\alpha})$ up to $t=104.8$ and $t=109$ observed in the simulation. $\mathbf{c}-\mathbf{h}$, Dynamics of the total density of infected hosts, mean antigenicity, mean virulence, variance in antigenicity, variance in virulence, and covariance between antigenicity and virulence, respectively predicted by the oligomorphic analysis compared to the simulation results. Parameters: $\gamma=0.5, \beta(\alpha)=5 \sqrt{\alpha}, D_{x}=0.005, D_{\alpha}$ $=0.0002$. As in Fig. 2, we assume a Gaussian cross-immunity kernel, $\sigma(x-y)=\exp \left(-(x-y)^{2} / 2 \omega^{2}\right)$, with width $\omega=5$. The oligomorphic dynamics describing the changes in the frequency $p_{0}(t)=1-p_{1}(t)$ of the currently prevailing morph at time $t_{s}=104.8$, the frequency $p_{1}(t)$ of the upcoming morph, the mean antigenicity $\bar{x}_{i}(t)$ and mean virulence $\bar{\alpha}_{i}(t)$ of the two morphs $(i=0,1)$, the within-morph variances $V_{i}^{x}$ and $V_{i}^{\alpha}$ in antigenicity and virulence, and the within-morph covariance $C_{i}(t)$ between antigenicity and virulence in each morph $(i=0,1)$ are defined as equations $(48)$ - $(53)$ in the methods.

how ecological/epidemiological dynamics can impact evolutionary outcomes that, in turn, feedback into the epidemiology characteristics of the disease.

We have used oligomorphic dynamics ${ }^{27}$ to make predictions on the waiting times and outcomes of the antigenic jumps in our model with cross immunity. This approach tracks changes in both mean trait values and trait variances in models with explicit ecological dynamics. As such, it combines aspects of eco-evolutionary theory $^{39,40}$ and quantitative genetics approaches ${ }^{41,42}$ to provide a more complete understanding of the evolution of quantitative traits. Our 
approach can take into account a wide range of different ecological and evolutionary timescales and therefore allows us to address fundamental questions on eco-evolutionary feedbacks and on the separation between ecological and evolutionary timescales. This is important since it allows us to test the implications of the different assumptions of classical evolutionary theory and to better understand the role of eco-evolutionary feedbacks on evolutionary outcomes. Furthermore, the approach can be applied widely to model transient dynamics, and to predict the waiting times and extent of diversification that occurs in a range of contexts ${ }^{27,43}$. Moreover, antigenic evolution is known to also lead to diversification and variant coexistence ${ }^{44-47}$, and it would be interesting to extend our analysis to these other evolutionary outcomes.

Our results emphasize that epidemiological dynamics may have important implications for the evolution of infectious disease. To facilitate its broader application, the oligomorphic methodology should be extended to structured populations and combined with stochastic evolutionary theory to fully address the evolutionary dynamics of emerging disease. Human coronaviruses can evolve antigenically to escape antibody immunity ${ }^{48}$, and it would be useful to apply our approaches to a more specific model of the Severe acute respiratory syndrome coronavirus 2 (SARS-Cov-2) epidemic. In particular, our ability to predict the waiting time until the emergence of the next antigenic cluster has the potential to be important in such applied contexts.

In principle, epidemics of new variants that adapt to a novel host would display equivalent dynamics to those described here for antigenic escape. It also follows that interventions that impact epidemiological dynamics may also have impacts on the evolution of pathogen traits, such as virulence or transmission. Our results suggest that immune escape driven by transmission blocking imperfect vaccination might also select for higher virulence in the longer term ${ }^{49,50}$, although these effects are probably overwhelmed by selection on transmission in an emerging pandemic such as SARS-CoV- $2^{51,52}$. Furthermore, dynamical feedbacks are important in a range of contexts beyond infectious disease, and our approach may help us examine the importance of interactions between frequency-dependent 'stabilizing' and equalizing evolutionary driv$\mathrm{ers}^{53}$. The oligomorphic analytical approaches we use here are therefore likely to be useful in understanding a wide range of dynamical evolutionary outcomes.

\section{Methods}

Oligomorphic dynamics (OMD) of antigenic escape. We considered a model of the antigenic escape of a pathogen from host herd immunity on a one-dimensional antigenicity space $(x)$. We tracked the changes in the density $S(t, x)$ of hosts that are susceptible to antigenicity variant $x$ of pathogen at time $t$, and the density $I(t, x)$ of hosts that are currently infected and infectious with antigenicity variant $x$ of pathogen at time $t$ :

$$
\begin{gathered}
\frac{\partial S(t, x)}{\partial t}=-S(t, x) \int_{-\infty}^{\infty} \beta \sigma(x-y) I(t, y) d y, \\
\frac{\partial I(t, x)}{\partial t}=\beta S(t, x) I(t, x)-(\gamma+\alpha) I(t, x)+D \frac{\partial^{2} I(t, x)}{\partial x^{2}},
\end{gathered}
$$

where $\beta, \alpha$ and $\gamma$ are the transmission rate, virulence (additional mortality due to infection) and recovery rate of pathogens, which are independent of antigenicity. The function $\sigma(x-y)$ denotes the degree of cross immunity: a host infected by pathogen variant $y$ acquires perfect cross immunity with probability $\sigma(x-y)$, but fails to acquire any cross immunity with probability $1-\sigma(x-y)$ (this is called polarized cross immunity by Gog and Grenfell $\left.{ }^{25}\right)$. The degree $\sigma(x-y)$ of cross immunity is assumed to be a decreasing function of the distance $|x-y|$ between variants $x$ and $y$. When a new variant with antigenicity $x=0$ is introduced at time $t=0$, the initial host population is assumed to be susceptible to any antigenicity variant of pathogen: $S(0, x)=1$. In equation (6), $D=\mu \sigma_{\mathrm{m}}^{2} / 2$ is one half of the mutation variance for the change in antigenicity, representing random mutation in the continuous antigenic space.

Susceptibility profile moulded by the primary outbreak. We first analysed the dynamics of the primary outbreak of a pathogen and derived the resulting susceptibility profile, which can be viewed as the fitness landscape subsequently experienced by the pathogen. For simplicity, we assumed that mutation can be ignored during the first epidemic initiated with antigenicity strain $x=0$. The density $S_{0}(t)=S(t, 0)$ of hosts that are susceptible to the currently prevailing antigenicity variant $x=0$, as well as the density $I_{0}(t)=I(t, 0)$ of hosts that are currently infected by the focal variant change with time as

$$
\begin{gathered}
\frac{d S_{0}}{d t}=-S_{0} \beta I_{0}, \\
\frac{d I_{0}}{d t}=S_{0} \beta I_{0}-(\gamma+\alpha) I_{0}, \\
\frac{d R_{0}}{d t}=\gamma I_{0},
\end{gathered}
$$

with $S_{0}(0)=1, I_{0}(0) \approx 0$ and $R_{0}(0)=0$. The final size of the primary outbreak,

$$
\psi_{0}=R_{0}(\infty)=1-S_{0}(\infty)=\exp \left[-\beta \int_{0}^{\infty} I_{0}(t) d t\right] \text {, }
$$

is determined as the unique positive root of

$$
\psi_{0}=1-e^{-\rho_{0} \psi_{0}},
$$

where $\rho_{0}=\beta /(\gamma+\alpha)>1$ is the basic reproductive number ${ }^{6}$. Associated with this epidemiological change, the susceptibility profile $S_{x}(t)=S(t, x)$ against antigenicity $x(x \neq 0)$ other than the currently circulating variant $(x=0)$ changes by cross immunity as

$$
\frac{d S_{x}}{d t}=-S_{x} \beta \sigma(x) I_{0}, \quad(x \neq 0) .
$$

Integrating both sides of equation (11) from $t=0$ to $t=\infty$, we see that the susceptibility profile $s(x)=S_{x}(\infty)$ after the primary outbreak at $x=0$ is

$$
s(x)=\exp \left[-\beta \sigma(x) \int_{0}^{\infty} I_{0}(t) d t\right]=\left(1-\psi_{0}\right)^{\sigma(x)}=e^{-\rho_{0} \sigma(x) \psi_{0}},
$$

where the last equality follows from equation (10). The susceptibility can be effectively reduced by cross immunity when the primary variant has a large impact (that is, when the fraction of hosts remaining uninfected, $1-\psi_{0}$, is small) and when the degree of cross immunity is strong (that is, when $\sigma(x)$ is close to 1 ). With a variant antigenically very close to the primary variant $(x \approx 0)$, the cross immunity is very strong $(\sigma(x) \approx 1)$ so that the susceptibility against variant $x$ is nearly maximally reduced: $s(x) \approx 1-\psi_{0}$. With a variant antigenically distant from the primary variant, $\sigma(x)$ becomes substantially smaller than 1 , making the host more susceptible to the variant. For example, if the cross immunity is halved $(\sigma(x)=0.5)$ from its maximum value 1 , then the susceptibility to that variant is as large as $\left(1-\psi_{0}\right)^{0.5}$. If a variant is antigenically very distant from the primary variant, then $\sigma(x) \approx 0$, and the host is nearly fully susceptible to the variant $(s(x) \approx 1)$.

\section{Threshold antigenic distance for escaping immunity raised by primary} outbreak. Of particular interest is the threshold antigenicity distance $x_{c}$ that allows for antigenic escape, that is, any antigenicity variant more distant than this threshold from the primary variant $\left(x>x_{c}\right)$ can increase when introduced after the primary outbreak. Such a threshold is determined from

$$
\frac{\beta s\left(x_{c}\right)}{\gamma+\alpha}=\rho_{0} s\left(x_{c}\right)=1
$$

or

$$
s\left(x_{c}\right)=\left(1-\psi_{0}\right)^{\sigma\left(x_{c}\right)}=e^{-\rho_{0} \sigma\left(x_{c}\right) \psi_{0}}=\frac{1}{\rho_{0}},
$$

where we used equation (12). With a specific choice of cross-immunity profile,

$$
\sigma(x)=\exp \left[-\frac{x^{2}}{2 \omega^{2}}\right],
$$

the threshold antigenicity beyond which the virus can increase in the susceptibility profile $s(x)$ after the primary outbreak is obtained, by substituting equation (14) into equation (13)

$$
\exp \left[-\rho_{0} \psi_{0} \exp \left[-\frac{x_{c}^{2}}{2 \omega^{2}}\right]\right]=\frac{1}{\rho_{0}},
$$

and taking the logarithm of both sides twice: 


$$
x_{c}=\omega \sqrt{2 \log \frac{\rho_{0} \psi_{0}}{\log \rho_{0}}} .
$$

OMD. Integrating both sides of equation (6) over the whole space, we obtained the dynamics for the total density of infected hosts, $\bar{I}(t)=\int_{-\infty}^{\infty} I(t, x) d x$ :

$$
\frac{d \bar{I}}{d t}=\left[\beta \int_{-\infty}^{\infty} S(t, x) \phi(t, x) d x-(\gamma+\alpha)\right] \bar{I}(t)=[\beta \bar{S}(t)-(\gamma+\alpha)] \bar{I}(t),
$$

where

$$
\phi(t, x)=I(t, x) / \bar{I}(t)
$$

is the relative frequency of antigenicity variant $x$ in the pathogen population circulating at time $t$, and

$$
\bar{S}(t)=\int_{-\infty}^{\infty} S(t, x) \phi(t, x) d x
$$

is the mean susceptibility experienced by currently circulating pathogens. The dynamics for the relative frequency $\phi(t, x)$ of pathogen antigenicity is

$$
\frac{\partial \phi}{\partial t}=\beta\{S(t, x)-\bar{S}(t)\} \phi(t, x)+D \frac{\partial^{2} \phi}{\partial x^{2}} .
$$

As in Sasaki and Dieckmann ${ }^{27}$, we decomposed the frequency distribution to the sum of several morph distributions (oligomorphic decomposition) as

$$
\phi(t, x)=\sum_{i} p_{i} \phi_{i}(t, x),
$$

where $p_{i}(t)$ is the frequency of morph $i$ and $\phi_{i}(t, x)$ is the within-morph distribution of antigenicity. By definition, and $\int_{-\infty}^{\infty} \phi_{i}(t, x) d x=1$. Let

$$
\bar{x}_{i}=\int_{-\infty}^{\infty} x \phi_{i}(t, x) d x
$$

be the mean antigenicity of a morph and

$$
V_{i}=\int_{-\infty}^{\infty}\left(x-\bar{x}_{i}\right)^{2} \phi_{i}(t, x) d x=O\left(\epsilon^{2}\right)
$$

where $O$ is order be the within-morph variance of each morph, which is assumed to be small, of the order of $\epsilon^{2}$. We denoted the mean susceptibility of host population for viral morph by $\bar{S}_{i}=\int_{-\infty}^{\infty} S(t, x) \phi_{i}(t, x) d x$. As shown in Sasaki and Dieckmann $^{27}$, the dynamics for viral morph frequency is expressed as

$$
\frac{d p_{i}}{d t}=\beta\left(\bar{S}_{i}-\bar{S}\right) p_{i}+O(\epsilon),
$$

while the dynamics for the within-morph distribution of antigenicity is

$$
\frac{\partial \phi_{i}}{\partial t}=\beta\left\{S(t, x)-\bar{S}_{i}\right\} \phi_{i}(t, x)+D \frac{\partial^{2} \phi_{i}}{\partial x^{2}} .
$$

From this, the dynamics for the mean antigenicity of a morph,

$$
\frac{d \bar{x}_{i}}{d t}=\left.V_{i} \beta \frac{\partial S}{\partial x}\right|_{x=\bar{x}_{i}}+O\left(\epsilon^{3}\right)
$$

and the dynamics for the within-morph variance of a morph

$$
\frac{d V_{i}}{d t}=\left.\frac{1}{2} \beta \frac{\partial^{2} S}{\partial x^{2}}\right|_{x=\bar{x}_{i}}\left\{E\left[\xi_{i}^{4}\right]-V_{i}^{2}\right\}+2 D+O\left(\epsilon^{5}\right)
$$

are derived, where $\xi_{i}=x-\bar{x}_{i}$ and $E\left[\xi_{i}^{4}\right]=\int^{\infty}\left(x-\bar{x}_{i}\right)^{4} \phi_{i}(t, x) d x$ are the fourth central moments of antigenicity around the morph mean. Assuming that the within-morph distribution is normal (Gaussian closure), $E\left[\xi_{i}^{4}\right]=3 V_{i}^{2}$, and hence equation (25) becomes

$$
\frac{d V_{i}}{d t}=\left.\beta \frac{\partial^{2} S}{\partial x^{2}}\right|_{x=\bar{x}_{i}} V_{i}^{2}+2 D+O\left(\epsilon^{5}\right) .
$$

Second outbreak predicted by OMD. Equations (22), (24) and (26) are general, but they rely on a full knowledge of the dynamics of the susceptibility profile $S(t, x)$. To make further progress, we used an additional approximation by substituting equation (13), the susceptibility profile, over viral antigenicity space after the primary outbreak at $x=0$ and before the onset of the second outbreak at a distant position. We kept track of two morphs at positions $x_{0}(t)$ and $x_{1}(t)$, where the first morph is that caused by the primary outbreak at $x=0$, and the second morph is that emerged in the range $x>x_{c}$ beyond the threshold antigenicity $x_{c}$ defined in equation (13) (and equation (15) for a specific form of $\sigma(x)$ ) as the source of the next outbreak.

$$
\text { As } \begin{aligned}
s(x)=\left(1-\psi_{0}\right)^{\sigma(x)} & =\exp \left[\sigma(x) \log \left(1-\psi_{0}\right)\right], \text { we have } \\
\frac{\mathrm{d} s}{\mathrm{~d} x}\left(\bar{x}_{i}\right) & =\left[\frac{\mathrm{d} \sigma}{\mathrm{d} x}\left(\bar{x}_{i}\right) \log \left(1-\psi_{0}\right)\right] s\left(\bar{x}_{i}\right),
\end{aligned}
$$

and

$$
\frac{\mathrm{d}^{2} s}{\mathrm{~d} x^{2}}\left(\bar{x}_{i}\right)=\left[\frac{\mathrm{d}^{2} \sigma}{\mathrm{d} x^{2}}\left(\bar{x}_{i}\right) \log \left(1-\psi_{0}\right)+\left\{\frac{\mathrm{d} \sigma}{\mathrm{d} x}\left(\bar{x}_{i}\right) \log \left(1-\psi_{0}\right)\right\}^{2}\right] s\left(\bar{x}_{i}\right) .
$$

Therefore, the frequency, mean antigenicity and variance of antigenicity of an emerging morph $(i=1)$ change respectively as

$$
\begin{aligned}
\frac{d p_{1}}{d t} & =\beta\left[s\left(\bar{x}_{1}\right)-s\left(\bar{x}_{0}\right)\right] p_{1}\left(1-p_{1}\right), \\
\frac{d \bar{x}_{1}}{d t} & =V_{1} \beta \frac{\mathrm{d} s}{\mathrm{~d} x}\left(\bar{x}_{1}\right), \\
\frac{d V_{1}}{d t} & =\beta \frac{\mathrm{d}^{2} s}{\mathrm{~d} x^{2}}\left(\bar{x}_{1}\right) V_{1}^{2}+2 D .
\end{aligned}
$$

The predicted change in the mean antigenicity was plotted by integrating equation (27). As initial condition, we chose the time when a seed of second peak in the range $x>x_{c}$ first appeared, and then computed the mean trait as

$$
\bar{x}(t)=x_{0}\left(1-p_{1}(t)\right)+\bar{x}_{1} p_{1}(t) .
$$

In the case of Fig. 2, where $\beta=2, \gamma+\alpha=0.6, D=0.001$ and $\omega=2$, the final size of epidemic for the primary outbreak, defined as equation (7), was $\psi=0.959$, and the critical antigenic distance for the increase of pathogen variant obtained from equation (26) was $x_{c}=2.795$. The initial conditions for the oligomorphic dynamics (equation 27) for the second morph were then $p_{1}\left(t_{0}\right)=1.6 \times 10^{-8}$, $\bar{x}_{1}\left(t_{0}\right)=3.239, V_{1}\left(t_{0}\right)=0.2675$ at $t_{0}=41$. In Fig. 2 , the predicted trajectory for the mean antigenicity (equation 28) is plotted as a red curve, together with the mean antigenicity change observed in simulation (blue curve).

Accuracy of predicting the antigenicity with OMD and the timing of the second outbreak. Here we describe how we defined the initial conditions for oligomorphic dynamics, that is, the frequency, the mean antigenicity and the variance in antigenicity of the morph that caused the primary outbreak and the morph that may cause the second outbreak. We then show how the accuracy in prediction of the second outbreak depends on the timing of the prediction.

We divided the antigenicity space into two at $x=x_{c}$, above which the pathogen can increase under the given susceptibility profile after the primary outbreak, but below which the pathogen cannot increase. We then took the relative frequencies of pathogens above $x_{c}$ and below $x_{c}$, and the conditional mean and variance in these separated regions to set the initial frequencies, means and variances of the morphs at time $t_{0}$ when we started integrating the oligomorphic dynamics to predict the second outbreak:

$$
\begin{array}{ll}
p_{0}\left(t_{0}\right)=\frac{\int_{0}^{x_{c}} I\left(t_{0}, x\right) d x}{\int_{0}^{\infty} I\left(t_{0}, x\right) d x}, & p_{1}\left(t_{0}\right)=\frac{\int_{x_{c}}^{\infty} I\left(t_{0}, x\right) d x}{\int_{0}^{\infty} I I\left(t_{0}, x\right) d x}, \\
\bar{x}_{0}\left(t_{0}\right)=\frac{\int_{0}^{x_{c}} x I\left(t_{0}, x\right) d x}{\int_{0}^{x_{c}} I\left(t_{0}, x\right) d x}, & \bar{x}_{1}\left(t_{0}\right)=\frac{\int_{x_{c}}^{\infty} x I\left(t_{0}, x\right) d x}{\int_{x_{c}}^{\infty} I\left(t_{0}, x\right) d x}, \\
V_{0}\left(t_{0}\right)=\frac{\int_{0}^{x_{c}}\left(x-\bar{x}_{0}\left(t_{0}\right)\right)^{2} I\left(t_{0}, x\right) d x}{\int_{0}^{x_{c}} I\left(t_{0}, x\right) d x}, & V_{1}\left(t_{0}\right)=\frac{\int_{x_{c}}^{\infty}\left(x-\bar{x}_{1}\left(t_{0}\right)\right)^{2} I\left(t_{0}, x\right) d x}{\int_{x_{c}}^{\infty} I\left(t_{0}, x\right) d x} .
\end{array}
$$

We then compared the trajectory for mean antigenicity change observed in simulation (blue curve in Fig. 2) and the predicted trajectory (red curve in Fig. 2) for mean antigenicity (equation 28) by integrating oligomorphic dynamics (equation 27) with the initial condition (equation 29) at time $t=t_{0}$. Extended Data Fig. 2 shows how the accuracy of prediction, measured by the Kullback-Leibler divergence between these two trajectories, depends on the timing $t_{0}$ chosen for the prediction. The second outbreak occurs around $t=54.6$, where mean antigenicity jumps from around 0 to around 5. The prediction with OMD is accurate if it is made for $t_{0}>40$. Figure 2 is drawn for $t_{0}=41$ where the second peak is about to emerge (see Extended Data Fig. 2). Even for the latest prediction for $t_{0}=51$ in Extended Data Fig. 2, the morph frequency of the emerging second morph was only $0.3 \%$ off, so the prediction is still worthwhile to make.

Extended Data Fig. 2 shows that the prediction power is roughly constant (albeit with a wiggle) for $5<t_{0}<30$ (the predicted timings are $10-15 \%$ longer than actual timing for $5<t_{0}<30$ ), and steadily improved for $t_{0}>30$. When the prediction was made very early $\left(t_{0}<5\right)$, the deviations were larger.

OMD for the joint evolution of antigenicity and virulence. Let $s(x)$ be the susceptibility of the host population against antigenicity $x$. A specific susceptibility profile is given by equation (12), with cross-immunity function $\sigma(x)$ and the final size $\psi_{0}$ of epidemic of the primary outbreak. Note that, as above, the susceptibility profile is, in general, a function of time. The density $I(x, \alpha)$ of hosts infected by a pathogen of antigenicity $x$ and virulence $\alpha$ changes with time, when rare, as

$$
\frac{\partial I(x, \alpha)}{\partial t}=\beta s(x) I(x, \alpha)-(\gamma+\alpha) I(x, \alpha)+D_{x} \frac{\partial^{2} I}{\partial x^{2}}+D_{\alpha} \frac{\partial^{2} I}{\partial \alpha^{2}} .
$$


The change in the frequency $\phi(x, \alpha)=I(x, \alpha) / \int I(x, \alpha) d x d \alpha$ of a pathogen with antigenicity $x$ and virulence $\alpha$ follows

$$
\frac{\partial \phi}{\partial t}=\{w(x, \alpha)-\bar{w}\} \phi+D_{x} \frac{\partial^{2} \phi}{\partial x^{2}}+D_{\alpha} \frac{\partial^{2} \phi}{\partial \alpha^{2}},
$$

where

$$
w(x, \alpha)=\beta(\alpha) s(x)-\alpha
$$

is the fitness of a pathogen with antigenicity $x$ and virulence $\alpha$ and $\bar{w}=\int w(x, \alpha) d x d \alpha$ is the mean fitness.

We decomposed the joint frequency distribution $\phi(x, \alpha)$ of the viral quasi-species as (oligomorphic decomposition):

$$
\phi(x, \alpha)=\sum_{i} \phi_{i}(x, \alpha) p_{i}
$$

where $\phi_{i}(x, \alpha)$ is the joint frequency distribution of antigenicity $x$ and virulence $\alpha$ in morph $i\left(\int \phi_{i} d x d \alpha=1\right)$ and $p_{i}$ is the relative frequency of morph $i\left(\sum_{i} p_{i}=1\right)$. The frequency of morph $i$ then changes as

$$
\begin{aligned}
& \frac{d p_{i}}{d t}=\left(\bar{w}_{i}-\sum_{j} \bar{w}_{j} p_{j}\right) p_{i}, \\
& \frac{\partial \phi_{i}}{\partial t}=\left(w(x, \alpha)-\bar{w}_{i}\right) \phi_{i}(x, \alpha)+D_{x} \frac{\partial^{2} \phi_{i}}{\partial x^{2}}+D_{\alpha} \frac{\partial^{2} \phi_{i}}{\partial \alpha^{2}},
\end{aligned}
$$

where $\bar{w}_{i}=\int w(x, \alpha) \phi_{i}(x, \alpha) d x d \alpha$ is the mean fitness of morph $i$.

Assuming that the traits are distributed narrowly around the morph means $\bar{x}_{i}=\int x \phi_{i}(x, \alpha) d x d \alpha$ and $\bar{\alpha}_{i}=\int \alpha \phi_{i}(x, \alpha) d x d \alpha$, so that $\xi_{i}=x-\bar{x}_{i}=O(\epsilon)$ and $\zeta_{i}=\alpha-\bar{\alpha}_{i}=O(\epsilon)$ where $\epsilon$ is a small constant, we expanded the fitness $w(x, \alpha)$ around the means $\bar{x}_{i}$ and $\bar{\alpha}_{i}$ of morph $i$,

$$
\begin{aligned}
w(x, \alpha)= & w\left(\bar{x}_{i}, \bar{\alpha}_{i}\right)+\left(\frac{\partial w}{\partial x}\right)_{i} \xi_{i}+\left(\frac{\partial w}{\partial \alpha}\right)_{i} \zeta_{i} \\
& +\frac{1}{2}\left(\frac{\partial^{2} w}{\partial x^{2}}\right)_{i} \xi_{i}^{2}+\left(\frac{\partial^{2} w}{\partial x \partial \alpha}\right)_{i} \xi_{i} \zeta_{i}+\frac{1}{2}\left(\frac{\partial^{2} w}{\partial \alpha^{2}}\right)_{i} \zeta_{i}^{2}+O\left(\epsilon^{3}\right) .
\end{aligned}
$$

Substituting this and

$$
\bar{w}_{i}=w\left(\bar{x}_{i}, \bar{\alpha}_{i}\right)+\frac{1}{2}\left(\frac{\partial^{2} w}{\partial x^{2}}\right)_{i} V_{i}^{x x}+\left(\frac{\partial^{2} w}{\partial x \partial \alpha}\right)_{i} V_{i}^{x \alpha}+\frac{1}{2}\left(\frac{\partial^{2} w}{\partial \alpha^{2}}\right)_{i} V_{i}^{\alpha \alpha}+O\left(\epsilon^{3}\right)
$$

into equation (34), we obtained

$$
\begin{gathered}
\frac{d p_{i}}{d t}=\left[w_{i}-\sum_{j} w_{j} p_{j}\right] p_{i}+O(\epsilon), \\
\frac{\partial \phi_{i}}{\partial t}=\left[\left(\frac{\partial w}{\partial x}\right)_{i} \xi_{i}+\left(\frac{\partial w}{\partial \alpha}\right)_{i} \zeta_{i}+\frac{1}{2}\left(\frac{\partial^{2} w}{\partial x^{2}}\right)_{i}\left(\xi_{i}^{2}-V_{i}^{x}\right)+\left(\frac{\partial^{2} w}{\partial x \partial \alpha}\right)_{i}\left(\xi_{i} \zeta_{i}-C_{i}\right)\right. \\
\left.+\frac{1}{2}\left(\frac{\partial^{2} w}{\partial \alpha^{2}}\right)_{i}\left(\zeta_{i}^{2}-V_{i}^{\alpha}\right)\right] \phi_{i}+D_{x} \frac{\partial^{2} \phi_{i}}{\partial x^{2}}+D_{\alpha} \frac{\partial^{2} \phi_{i}}{\partial \alpha^{2}}+O\left(\epsilon^{3}\right),
\end{gathered}
$$

where $w_{i}=w\left(\bar{x}_{i}, \bar{\alpha}_{i}\right),\left(\frac{\partial w}{\partial x}\right)_{i}=\frac{\partial w}{\partial x}\left(\bar{x}_{i}, \bar{\alpha}_{i}\right),\left(\frac{\partial w}{\partial \alpha}\right)_{i}=\frac{\partial w}{\partial \alpha}\left(\bar{x}_{i}, \bar{\alpha}_{i}\right)$,

$\left(\frac{\partial^{2} w}{\partial x^{2}}\right)_{i}=\frac{\partial^{2} w}{\partial x^{2}}\left(\bar{x}_{i}, \bar{\alpha}_{i}\right),\left(\frac{\partial^{2} w}{\partial x \partial \alpha}\right)_{i}=\frac{\partial^{2} w}{\partial x \partial \alpha}\left(\bar{x}_{i}, \bar{\alpha}_{i}\right)$ and $\left(\frac{\partial^{2} w}{\partial \alpha^{2}}\right)_{i}=\frac{\partial^{2} w}{\partial \alpha^{2}}\left(\bar{x}_{i}, \bar{\alpha}_{i}\right)$ are fitness and its first and second derivatives evaluated at the mean traits of morph $i$, and

$$
\begin{aligned}
& V_{i}^{x}=E_{i}\left[\left(x-\bar{x}_{i}\right)^{2}\right], \\
& C_{i}=E_{i}\left[\left(x-\bar{x}_{i}\right)\left(\alpha-\bar{\alpha}_{i}\right)\right], \\
& V_{i}^{\alpha}=E_{i}\left[\left(\alpha-\bar{\alpha}_{i}\right)^{2}\right]
\end{aligned}
$$

are within-morph variances and covariance of the traits of morph $i$. Here $E_{i}[f(x, \alpha)]=\int f(x, \alpha) \phi_{i}(x, \alpha) d x d \alpha$ denotes taking expectation of a function $f$ with respect to the joint trait distribution $\phi_{i}(x, \alpha)$ of morph $i$.

Substituting equation (36) into the change in the mean antigenicity of morph $i$

$$
\frac{d \bar{x}_{i}}{d t}=\frac{d}{d t} \iint x \phi_{i}(x, \alpha) d x d \alpha=\iint x \frac{\partial \phi_{i}}{\partial t} d x d \alpha=\iint\left(\bar{x}_{i}+\xi_{i}\right) \frac{\partial \phi_{i}}{\partial t} d \xi_{i} d \zeta_{i},
$$

we obtained

$$
\frac{d \bar{x}_{i}}{d t}=\left(\frac{\partial w}{\partial x}\right)_{i} V_{i}^{x}+\left(\frac{\partial w}{\partial \alpha}\right)_{i} C_{i}+O\left(\epsilon^{3}\right)
$$

Similarly, the change in the mean virulence of morph $i$ was expressed as

$$
\frac{d \bar{\alpha}_{i}}{d t}=\left(\frac{\partial w}{\partial x}\right)_{i} C_{i}+\left(\frac{\partial w}{\partial \alpha}\right)_{i} V_{i}^{\alpha}+O\left(\epsilon^{3}\right) .
$$

Equations (38) and (39) from the mean trait change was summarized in a matrix form as

$$
\frac{d}{d t}\left(\begin{array}{c}
\bar{x}_{i} \\
\bar{\alpha}_{i}
\end{array}\right)=\boldsymbol{G}_{i}\left(\begin{array}{c}
\left(\frac{\partial w}{\partial x}\right)_{i} \\
\left(\frac{\partial w}{\partial \alpha}\right)_{i}
\end{array}\right)+O\left(\epsilon^{3}\right)
$$

where

$$
\boldsymbol{G}_{\boldsymbol{i}}=\left(\begin{array}{cc}
V_{i}^{x} & C_{i} \\
C_{i} & V_{i}^{\alpha}
\end{array}\right)
$$

is the variance-covariance matrix of the morph $i$.

Substituting equation (36) into the right-hand side of the change in variance of antigenicity of morph $i$,

$$
\frac{d V_{i}^{x}}{d t}=\frac{d}{d t} \iint \xi_{i}^{2} \phi_{i} d \xi_{i} d \zeta_{i}=\iint \xi_{i}^{2} \frac{\partial \phi_{i}}{\partial t} d \xi_{i} d \zeta_{i}
$$

and those in the change in the other variance and covariance, we obtained

$$
\begin{aligned}
\frac{d V_{i}^{x}}{d t}= & \frac{1}{2}\left(\frac{\partial^{2} w}{\partial x^{2}}\right)_{i}\left[E_{i}\left(\xi_{i}^{4}\right)-\left(V_{i}^{x}\right)^{2}\right]+\left(\frac{\partial^{2} w}{\partial x \partial \alpha}\right)_{i}\left[E_{i}\left(\xi_{i}^{3} \zeta_{i}\right)-V_{i}^{x} C_{i}\right] \\
& +\frac{1}{2}\left(\frac{\partial^{2} w}{\partial \alpha^{2}}\right)_{i}\left[E_{i}\left(\xi_{i}^{2} \zeta_{i}^{2}\right)-V_{i}^{x} V_{i}^{\alpha}\right]+2 D_{x}+O\left(\epsilon^{5}\right), \\
\frac{d C_{i}}{d t}= & \frac{1}{2}\left(\frac{\partial^{2} w}{\partial x^{2}}\right)_{i}\left[E_{i}\left(\xi_{i}^{3} \zeta_{i}\right)-V_{i}^{x} C_{i}\right]+\left(\frac{\partial^{2} w}{\partial x \partial \alpha}\right)_{i}\left[E_{i}\left(\xi_{i}^{2} \zeta_{i}^{2}\right)-C_{i}^{2}\right] \\
& +\frac{1}{2}\left(\frac{\partial^{2} w}{\partial \alpha^{2}}\right)_{i}\left[E_{i}\left(\xi_{i} \zeta_{i}^{3}\right)-C_{i} V_{i}^{\alpha}\right]+O\left(\epsilon^{5}\right), \\
\frac{d V_{i}^{\alpha}}{d t}= & \frac{1}{2}\left(\frac{\partial^{2} w}{\partial x^{2}}\right)_{i}\left[E_{i}\left(\xi_{i}^{2} \zeta_{i}^{2}\right)-V_{i}^{x} V_{i}^{\alpha}\right]+\left(\frac{\partial^{2} w}{\partial x \partial \alpha}\right)_{i}\left[E_{i}\left(\xi_{i} \zeta_{i}^{3}\right)-C_{i} V_{i}^{\alpha}\right] \\
& +\frac{1}{2}\left(\frac{\partial^{2} w}{\partial \alpha^{2}}\right)_{i}\left[E_{i}\left(\zeta_{i}^{4}\right)-\left(V_{i}^{\alpha}\right)^{2}\right]+2 D_{\alpha}+O\left(\epsilon^{5}\right) .
\end{aligned}
$$

If we assume that antigenicity and virulence within a morph follow a $2 \mathrm{D}$ Gaussian distribution for given means, variances and covariance, we should have $E_{i}\left(\xi_{i}^{4}\right)=3\left(V_{i}^{x}\right)^{2}, E_{i}\left(\xi_{i}^{3} \zeta_{i}\right)=3 V_{i}^{x} C_{i}, E_{i}\left(\xi_{i}^{2} \zeta_{i}^{2}\right)=V_{i}^{x} V_{i}^{\alpha}+2 C_{i}^{2}, E_{i}\left(\xi_{i} \zeta_{i}^{3}\right)=3 V_{i}^{\alpha} C_{i}$ and $E_{i}\left(\zeta_{i}^{4}\right)=3\left(V_{i}^{\alpha}\right)^{2}$, and hence

$$
\frac{d V_{i}^{x}}{d t}=\left(\frac{\partial^{2} w}{\partial x^{2}}\right)_{i}\left(V_{i}^{x}\right)^{2}+2\left(\frac{\partial^{2} w}{\partial x \partial \alpha}\right)_{i} V_{i}^{x} C_{i}+\left(\frac{\partial^{2} w}{\partial \alpha^{2}}\right)_{i} C_{i}^{2}+2 D_{x}+O\left(\epsilon^{5}\right),
$$

$$
\frac{d C_{i}}{d t}=\left(\frac{\partial^{2} w}{\partial x^{2}}\right)_{i} V_{i}^{x} C_{i}+\left(\frac{\partial^{2} w}{\partial x \partial \alpha}\right)_{i}\left\{V_{i}^{x} V_{i}^{\alpha}-C_{i}^{2}\right\}+\left(\frac{\partial^{2} w}{\partial \alpha^{2}}\right)_{i} C_{i} V_{i}^{\alpha}+O\left(\epsilon^{5}\right),
$$

$$
\frac{d V_{i}^{\alpha}}{d t}=\left(\frac{\partial^{2} w}{\partial x^{2}}\right)_{i} C_{i}^{2}+2\left(\frac{\partial^{2} w}{\partial x \partial \alpha}\right)_{i} V_{i}^{\alpha} C_{i}+\left(\frac{\partial^{2} w}{\partial \alpha^{2}}\right)_{i}\left(V_{i}^{\alpha}\right)^{2}+2 D_{\alpha}+O\left(\epsilon^{5}\right) .
$$

Equations (43) and (44) were rewritten in a matrix form as

$$
\frac{d G_{i}}{d t}=G_{i} H_{i} G_{i}+\left(\begin{array}{cc}
2 D_{x} V_{i}^{x} & 0 \\
0 & 2 D_{\alpha} V_{i}^{\alpha}
\end{array}\right)+O\left(\epsilon^{5}\right),
$$

where

$$
H_{i}=\left(\begin{array}{cc}
\left(\frac{\partial^{2} w}{\partial x^{2}}\right)_{i} & \left(\frac{\partial^{2} w}{\partial x \partial \alpha}\right)_{i} \\
\left(\frac{\partial^{2} w}{\partial x \partial \alpha}\right)_{i} & \left(\frac{\partial^{2} w}{\partial \alpha^{2}}\right)_{i}
\end{array}\right),
$$

is the Hessian of the fitness function of morph $i$.

In our equation (30) of the joint evolution of antigenicity and virulence of a pathogen after its primary outbreak, the fitness function is given by $w(x, \alpha)=\beta(\alpha) s(x)-\alpha$, and hence $w_{i}=\beta\left(\bar{\alpha}_{i}\right) s\left(\bar{x}_{i}\right)-\bar{\alpha}_{i},\left(\frac{\partial w}{\partial x}\right)_{i}=\beta\left(\bar{\alpha}_{i}\right) s \prime\left(\bar{x}_{i}\right)$, $\left(\frac{\partial w}{\partial \alpha}\right)_{i}=\beta \prime\left(\bar{\alpha}_{i}\right) s\left(\bar{x}_{i}\right)-1,\left(\frac{\partial^{2} w}{\partial x^{2}}\right)_{i}=\beta\left(\bar{\alpha}_{i}\right) s \prime \prime\left(\bar{x}_{i}\right),\left(\frac{\partial^{2} w}{\partial x \partial \alpha}\right)_{i}=\beta \prime\left(\bar{\alpha}_{i}\right) s \prime\left(\bar{x}_{i}\right)$, $\left(\frac{\partial^{2} w}{\partial x \partial \alpha}\right)_{i}=\beta \prime\left(\bar{\alpha}_{i}\right) s \prime\left(\bar{x}_{i}\right)$ and $\left(\frac{\partial^{2} w}{\partial \alpha^{2}}\right)_{i}=\beta \prime \prime\left(\bar{\alpha}_{i}\right) s\left(\bar{x}_{i}\right)$, where a prime on $\beta(\alpha)$ and $s(x)$ denotes differentiation by $\alpha$ and $x$, respectively. Substituting these into the dynamics for morph frequencies (equation 35), for morph means (equations 38 and 39), and for within-morph variance and covariance (equations 43-45), we obtained 


$$
\begin{aligned}
& \frac{d p_{i}}{d t}=\left[\beta\left(\bar{\alpha}_{i}\right) s\left(\bar{x}_{i}\right)-\bar{\alpha}_{i}-\sum_{j}\left(\beta\left(\bar{\alpha}_{j}\right) s\left(\bar{x}_{j}\right)-\bar{\alpha}_{j}\right) p_{j}\right] p_{i}, \\
& \frac{d \bar{x}_{i}}{d t}=\beta\left(\bar{\alpha}_{i}\right) s \prime\left(\bar{x}_{i}\right) V_{i}^{x}+\left\{\beta \prime\left(\bar{\alpha}_{i}\right) s\left(\bar{x}_{i}\right)-1\right\} C_{i}, \\
& \frac{d \bar{\alpha}_{i}}{d t}=\beta\left(\bar{\alpha}_{i}\right) s \prime\left(\bar{x}_{i}\right) C_{i}+\left\{\beta \prime\left(\bar{\alpha}_{i}\right) s\left(\bar{x}_{i}\right)-1\right\} V_{i}^{\alpha}, \\
& \frac{d V_{i}^{x}}{d t}=\beta\left(\bar{\alpha}_{i}\right) s \prime \prime\left(\bar{x}_{i}\right)\left(V_{i}^{x}\right)^{2}+2 \beta \prime\left(\bar{\alpha}_{i}\right) s^{\prime}\left(\bar{x}_{i}\right) V_{i}^{x} C_{i}+\beta \prime \prime\left(\bar{\alpha}_{i}\right) s\left(\bar{x}_{i}\right) C_{i}^{2}+2 D_{x}, \\
& \frac{d C_{i}}{d t}=\beta\left(\bar{\alpha}_{i}\right) s \prime \prime\left(\bar{x}_{i}\right) V_{i}^{x} C_{i}+\beta \prime\left(\bar{\alpha}_{i}\right) s \prime\left(\bar{x}_{i}\right)\left\{V_{i}^{x} V_{i}^{\alpha}-C_{i}^{2}\right\}+\beta \prime \prime\left(\bar{\alpha}_{i}\right) s\left(\bar{x}_{i}\right) C_{i} V_{i}^{\alpha}, \\
& \frac{d V_{i}^{\alpha}}{d t}=\beta\left(\bar{\alpha}_{i}\right) s \prime \prime\left(\bar{x}_{i}\right) C_{i}^{2}+2 \beta \prime\left(\bar{\alpha}_{i}\right) s \prime\left(\bar{x}_{i}\right) V_{i}^{\alpha} C_{i}+\beta \prime \prime\left(\bar{\alpha}_{i}\right) s\left(\bar{x}_{i}\right)\left(V_{i}^{\alpha}\right)^{2}+2 D_{\alpha} .
\end{aligned}
$$

Equations (48)-(53) describe the oligomorphic dynamics of the joint evolution of antigenicity and virulence of a pathogen for a given host susceptibility profile $s(x)$ over pathogen antigenicity.

Of particular interest is whether antigenicity or virulence evolve faster when they jointly evolve than when they evolve alone. After the primary outbreak at a given antigenicity, for example $x=0$, the susceptibility $s(x)$ of the host population increases due to cross immunity as the distance $x>0$ from the antigenicity at the primary outbreak increases. Hence, $s \prime\left(\bar{x}_{i}\right)>0$. Combining this with the positive trade-off between transmission rate and virulence, we see that $\left(\partial^{2} w / \partial x \partial \alpha\right)_{i}=\beta \prime\left(\bar{\alpha}_{i}\right) s /\left(\bar{x}_{i}\right)>0$, and then from equation (52), we see that the within-morph covariance between antigenicity and virulence becomes positive starting from a zero initial value:

$$
\left.\frac{d C_{i}}{d t}\right|_{C_{i}=0}=\left(\frac{\partial^{2} w}{\partial x \partial \alpha}\right)_{i} V_{i}^{x} V_{i}^{\alpha}>0 .
$$

If all second moments are initially sufficiently small for an emerging morph, a quick look at the linearization of equations (51)-(53) around $\left(V_{i}^{x}, C_{i}, V_{i}^{\alpha}\right)=(0,0,0)$ indicates that both $V_{i}^{x}$ and $V_{i}^{\alpha}$ become positive due to the random generation of variance by mutation, $D_{x}>0$ and $D_{\alpha}>0$, while the covariance stays close to zero. Then, equation (54) guarantees that the first move of the covariance is from zero to positive, which then guarantees that $C_{i}>0$ for all $t$. Therefore, the second term in equation (38) is positive until the mean virulence reaches its optimum $(\beta \prime(\alpha) s(x)=1)$. This means that joint evolution with virulence accelerates the evolution of antigenicity. The same is true for virulence evolution: the first term in equation (39) (which denotes the associated change in virulence due to the selection in antigenicity through genetic covariance between them) is positive, indicating that joint evolution with antigenicity accelerates virulence evolution.

Numerical example. Figure 5 shows the oligomorphic dynamics prediction of the emergence of the next variant in antigenicity-virulence coevolution. To make progress numerically, we assumed $s(x)$ to be constant in the following analysis because we are interested in the process between the end of the primary outbreak and the emergence of the next antigenicity-virulence morph. The partial differential equations for the density of host $S(t, x)$ susceptible to the antigenicity variant $x$ at time $t$, and the density of hosts infected by the pathogen variant with antigenicity $x$ and virulence $\alpha$ are

$$
\begin{aligned}
& \frac{\partial S(t, x)}{\partial t}=-S(t, x) \int_{\alpha_{\min }}^{\alpha_{\max }} \int_{0}^{x_{\max }} \beta(\alpha) \sigma(x-y) I(t, y, \alpha) d y d \alpha, \\
& \frac{\partial I(t, x, \alpha)}{\partial t}=[\beta(\alpha) S(t, x)-(\gamma+\alpha)] I(t, x, \alpha)+\left(D_{x} \frac{\partial^{2}}{\partial x^{2}}+D_{\alpha} \frac{\partial^{2}}{\partial \alpha^{2}}\right) I(t, x, \alpha),
\end{aligned}
$$

with the boundary conditions

$(\partial S / \partial x)(t, 0)=(\partial S / \partial x)\left(t, x_{\max }\right)=0,(\partial \mathrm{I} / \partial x)(t, 0, \alpha)=(\partial \mathrm{I} / \partial x)\left(t, x_{\max }, 0\right)=0$, $(\partial I / \partial x)\left(t, x, \alpha_{\min }\right)=(\partial I / \partial x)\left(t, x, \alpha_{\max }\right)=0$, and the initial conditions $S(0, x)=1$ and $I(0, x, \alpha)=\epsilon \delta(x) \delta(\alpha)$, where $\delta(\cdot)$ is the delta function and $\epsilon=0.01$. The trait space is restricted in a rectangular region: $0<x<x_{\max }=300$ and $\alpha_{\min }=0.025<\alpha<10=\alpha_{\max }$. Oligomorphic dynamics prediction for the joint evolution of antigenicity and virulence was applied for the next outbreak after the outbreak with the mean antigenicity around $x=108$ at time $t=102$. The susceptibility of the host to antigenicity variant $x$ at $t_{0}=104.8$ after the previous outbreak peaked around time $t=102$ came to an end is

$$
s(x)=S\left(t_{0}, x\right) .
$$

This susceptibility profile remained unchanged until the next outbreak started, and hence the fitness of a pathogen variant with antigenicity $x$ and virulence $\alpha$ is given by

$$
w(x, \alpha)=\beta(\alpha) s(x)-(\gamma+\alpha) .
$$

We bundled the pathogen variants into two morphs at time $t_{0}$ at the threshold antigenicity $x_{c}$, above which the net growth rate of the pathogen variant under the given susceptibility profile $s(x)$ and the mean antigenicity become positive:

$$
w\left(x_{c}, \bar{\alpha}\left(t_{0}\right)\right)=\beta\left(\bar{\alpha}\left(t_{0}\right)\right) s\left(x_{c}\right)-\left(\gamma+\bar{\alpha}\left(t_{0}\right)\right)=0 .
$$

The initial frequency and the moments of the two morphs, the variant 0 with $x<x_{c}$ and the variant 1 with $x>x_{c}$ were then calculated respectively from the joint distribution $I\left(t_{0}, x, \alpha\right)$ in the restricted region $\left\{(x, \alpha) ; 0<x<x_{c}, \alpha_{\min }<\alpha<\alpha_{\max }\right\}$ and that in the restricted region $\left\{(x, \alpha) ; x_{c}<x<x_{\max }, \alpha_{\min }<\alpha<\alpha_{\max }\right\}$. The frequency $p_{1}$ of morph 1 (the frequency of morph 0 is given by $p_{0}=1-p_{1}$ ), the mean antigenicity $\bar{x}_{i}$ and mean virulence $\bar{\alpha}_{i}$ of morph $i$, and the variances and covariance, $V_{i}^{\alpha}$, and $V_{i}^{\alpha} C_{i}$ of morph $i(i=0,1)$ follow equations (48)-(53), where the dynamics for the morph frequency (equation 48) is simplified in this two-morph situation as

$$
\frac{d p_{1}}{d t}=\left[\beta\left(\bar{\alpha}_{1}\right) s\left(\bar{x}_{1}\right)-\beta\left(\bar{\alpha}_{0}\right) s\left(\bar{x}_{0}\right)-\left(\bar{\alpha}_{1}-\bar{\alpha}_{0}\right)\right] p_{1}\left(1-p_{1}\right),
$$

with $p_{0}(t)=1-p_{1}(t)$. This is iterated from $t=t_{0}=104.8$ to $t_{e}=107$. The frequency $p_{1}$ of the new morph, the population mean antigenicity $\bar{x}=p_{0} \bar{x}_{0}+p_{1} \bar{x}_{1}$, virulence $\bar{\alpha}=p_{0} \bar{\alpha}_{0}+p_{1} \bar{\alpha}_{1}$, variance in antigenicity $V_{x}=p_{0} V_{0}^{x}+p_{1} V_{1}^{x}$, covariance between antigenicity and virulence $C=p_{0} C_{0}+p_{1} C_{1}$, and variance in virulence $V_{\alpha}=p_{0} V_{0}^{\alpha}+p_{1} V_{1}^{\alpha}$ are overlayed by red thick curves on the trajectories of moments observed in the full dynamics (equation 55).

In Fig. $5 \mathrm{a}$, the dashed vertical line represents the threshold antigenicity $x_{c}$, above which $R_{0}=\beta s(x) /(\gamma+\bar{\alpha})>1$ at $t=t_{s}=104.8$, where oligomorphic dynamics prediction was attempted. Two morphs were then defined according to whether or not the antigenicity exceeded a threshold $x=x_{i}$ : the resident morph (morph 1) is represented as the dense cloud to the left of $x=x_{c}$ and the second morph (morph 2) consisting of all the genotypes to the right of $x=x_{c}$ with their $R_{0}$ greater than one. The within-morph means and variances were then calculated in each region. The relative total densities of infected hosts in the left and right regions defined the initial frequency of two morphs in OMD. A 2D Gaussian distribution was assumed for within-morph trait distributions to have the closed moment equations as previously explained. Using these as the initial means, variances, covariances of the two morphs at $t=t_{s}$, the oligomorphic dynamics for 11 variables (relative frequency of morph 1 , mean antigenicity, mean virulence, variances in antigenicity and virulence and their covariance in morphs 0 and 1) was integrated up to $t=t_{e}$. The results are shown as red curves in Fig. $5 \mathrm{c}-\mathrm{h}$, which are compared with the simulation results (blue curves).

Fig. $5 \mathrm{c}-\mathrm{e}$ respectively show the change in total infected density, mean antigenicity and mean virulence. Red curves show the predictions by oligomorphic dynamics from the initial moments of each morph at $t=t_{s}$ to the susceptibility distribution $s(x)=S\left(t_{s}, x\right)$, which are compared with the simulation results (blue curves). The OMD-predicted mean antigenicity, for example, is defined as

$$
\bar{x}(t)=\left(1-p_{1}(t)\right) \bar{x}_{0}(t)+p_{1}(t) \bar{x}_{1}(t),
$$

where $p_{1}(t)$ is the frequency of morph $1, \bar{x}_{0}$ and $\bar{x}_{1}$ are the mean antigenicities of morphs 0 and 1 .

The red curves in Fig. $5 f-h$ show the OMD-predicted changes in the variance in antigenicity, variance in virulence and covariance between antigenicity and virulence, which are compared with the simulation results (blue curves). The OMD-predicted covariance, for example, is defined as

$$
\begin{aligned}
C(t)= & \left(1-p_{1}(t)\right) C_{0}(t)+p_{1}(t) C_{1}(t)+p_{1}(t)\left(1-p_{1}(t)\right) \\
& \left(\bar{x}_{0}(t)-\bar{x}_{1}(t)\right)\left(\bar{\alpha}_{0}(t)-\bar{\alpha}_{1}(t)\right),
\end{aligned}
$$

where $C_{0}(t)$ and $C_{1}(t)$ are the antigenicity-virulence covariances in morphs 0 and 1 , and $\bar{\alpha}_{0}(t)$ and $\bar{\alpha}_{1}(t)$ are the mean virulence of morphs 0 and 1 .

Selection for maximum growth rate. We next show that a pathogen that has the strategy of maximizing growth rate in a fully susceptible population is evolutionarily stable in the presence of antigenic escape.

At stationarity, the travelling wave profiles of $\hat{I}(z)$ and $\hat{S}(z)$ along the moving coordinate, $z=x-v t$, that drifts constantly to the right with the speed $v$ are defined as 


$$
\begin{aligned}
& 0=D \frac{d^{2} \hat{I}(z)}{d z^{2}}+v \frac{d \hat{I}(z)}{d z}+\beta \hat{S}(z) \hat{I}(z)-(\gamma+\alpha) \hat{I}(z), \\
& 0=v \frac{d \hat{S}(z)}{d z}-\beta \hat{S}(z) \int_{-\infty}^{\infty} \sigma(z-\xi) \hat{I}(\xi) d \xi,
\end{aligned}
$$

with $\hat{I}(-\infty)=\hat{I}(\infty)=0, \hat{S}(\infty)=1$.

Let $j(t, x)$ be the density of a mutant pathogen variant, with virulence $\alpha^{\prime}$ and transmission rate $\beta^{\prime}$, that is introduced in the host population where the resident variant is already established (equation 50). For the initial transient phase in which the density of mutants is sufficiently small, we have an equation for the change in $J(t, z)=j(t, x)$

$$
\frac{\partial}{\partial t} J(t, z)=\left\{D \frac{\partial^{2}}{\partial z^{2}}+v \frac{\partial}{\partial z}+\beta / \hat{S}(z)-(\gamma+\alpha \prime)\right\} J(t, z),
$$

with the initial condition $J(0, z)=\epsilon \delta(z)$, where $\epsilon$ is a small constant and $\delta(\cdot)$ is Dirac's function.

Consider a system

$$
\frac{\partial w}{\partial t}=\left\{D \frac{\partial^{2}}{\partial z^{2}}+v \frac{\partial}{\partial z}+\beta \prime-(\gamma+\alpha \prime)\right\} w,
$$

with $w(0, z)=J(0, z)=\epsilon \delta(z)$. Noting that $\hat{S}(z)<1$, we have $J(t, z) \leq w(t, z)$ for any $t>0$ and $z \in \mathbb{R}$ from the comparison theorem. The solution to equation (52) is

$$
w(t, z)=\frac{\epsilon}{\sqrt{4 \pi D t}} \exp \left[r / t-\frac{(z+v t)^{2}}{4 D t}\right],
$$

where $r \prime=\beta \prime-(\gamma+\alpha \prime)$. This follows by noting that $w(t, x) e^{-r \prime t}$ follows a simple diffusion equation $\partial w / \partial t=D \partial^{2} w / \partial x^{2}$. By rearranging the exponents of equation (53),

$$
\begin{aligned}
w(t, z)= & \exp [a t-k z] \frac{\epsilon}{\sqrt{4 \pi D t}} e^{-z^{2} / 4 D t} \\
& <\frac{\epsilon}{\sqrt{4 \pi D t}} \exp [a t-k z],
\end{aligned}
$$

where

$$
\begin{gathered}
a=\frac{v^{\prime 2}-v^{2}}{4 D}, \\
k=\frac{v}{2 D} .
\end{gathered}
$$

Here $v \prime=2 \sqrt{r \prime D}$ is the asymptotic wave speed if the mutant variant monopolizes the host population. Therefore, if $v^{\prime}<v$, then $a<0$, and hence $w(t, z)$ for a fixed $z$ converges to zero as $t$ goes to infinity; this, in turn, implies that $J(t, z)$ converges to zero because $J(t, z) \leq w(t, z)$ for all $t$ and $z$. Therefore, we conclude that any mutant that has a slower wave speed than the resident can never invade the population, implying that a variant that has the maximum wave speed $v=2 \sqrt{r D}$ is locally evolutionarily stable.

Reporting Summary. Further information on research design is available in the Nature Research Reporting Summary linked to this article.

\section{Code availability}

All code is available at https://github.com/MikeBoots/AntigenicEscape.

Received: 9 March 2021; Accepted: 28 October 2021;

Published online: 23 December 2021

\section{References}

1. Fauci, A. S. \& Morens, D. M. Zika virus in the Americas-yet another arbovirus threat. N. Engl. J. Med. 374, 601-604 (2016).

2. Coronavirus Disease 2019 (COVID-19): Situation Report, 72 (WHO, 2020).

3. Remuzzi, A. \& Remuzzi, G. COVID-19 and Italy: what next? The Lancet 395 , $1225-1228$ (2020)

4. Keeling, M. J. et al. Dynamics of the 2001 UK foot and mouth epidemic: stochastic dispersal in a heterogeneous landscape. Science 294, 813-817 (2001).

5. Hudson, P., Dobson, A., \& Newborn, D. Prevention of population cycles by parasite removal. Science 282, 2256-2258 (1998).

6. Anderson, R. M. \& May, R. M. Infectious Diseases of Humans (Oxford Univ Press, 1991).

7. May, R. M. \& Anderson, R. M. Parasite-host coevolution. Parasitology 100, S89-S101 (1990).

8. Bremermann, H. J. \& Pickering, J. A game-theoretical model of parasite virulence. J. Theor. Biol. 100, 411-426 (1983).
9. Alizon, S., Hurford, A., Mideo, N. \& van Baalen, M. Virulence evolution and the trade-off hypothesis: history, current state of affairs and the future. J. Evol. Biol. 22, 245-259 (2009).

10. Bremermann, H. J. \& Thieme, H. R. A competitive-exclusion principle for pathogen virulence. J. Math. Biol. 27, 179-190 (1989).

11. Alizon, S., de Roode, J. C. \& Michalakis, Y. Multiple infections and the evolution of virulence. Ecol. Lett. 16, 556-567 (2013).

12. Lion, S. \& Boots, M. Are parasites 'prudent'in space? Ecol. Lett. 13, 1245-1255 (2010).

13. van Baalen, M. Coevolution of recovery ability and virulence. Proc. R. Soc. Lond. B 265, 317-325 (1998).

14. Lion, S. \& Metz, J. A. Beyond R0 maximisation: on pathogen evolution and environmental dimensions. Trends Ecol. Evol. 33, 458-473 (2018).

15. Day, T. \& Gandon, S. Applying population-genetic models in theoretical evolutionary epidemiology. Ecol. Lett. 10, 876-888 (2007).

16. Day, T. \& Proulx, S. R. A general theory for the evolutionary dynamics of virulence. Am. Nat. 163, E40-E63 (2004).

17. Frank, S. A. Ecological and genetic models of host-pathogen coevolution. Heredity 67, 73-83 (1991).

18. Lenski, R. E. \& May, R. M. The evolution of virulence in parasites and pathogens - reconciliation between 2 competing hypotheses. J. Theor. Biol. 169, 253-265 (1994).

19. Archetti, I. \& Horsfall, F. L. Jr Persistent antigenic variation of influenza A viruses after incomplete neutralization in ovo with heterologous immune serum. J. Exp. Med. 92, 441-462 (1950).

20. Smith, D. J. et al. Mapping the antigenic and genetic evolution of influenza virus. Science 305, 371-376 (2004).

21. Hensley, S. E. et al. Hemagglutinin receptor binding avidity drives influenza A virus antigenic drift. Science 326, 734-736 (2009).

22. Gupta, S. et al. The maintenance of strain structure in populations of recombining infectious agents. Nat. Med. 2, 437-442 (1996).

23. Haraguchi, Y. \& Sasaki, A. Evolutionary pattern of intra-host pathogen antigenic drift: effect of cross-reactivity in immune response. Philos. Trans. $R$. Soc. Lond. B 352, 11-20 (1997).

24. Sasaki, A. \& Haraguchi, Y. Antigenic drift of viruses within a host: a finite site model with demographic stochasticity. J. Mol. Evol. 51, 245-255 (2000).

25. Gog, J. R. \& Grenfell, B. T. Dynamics and selection of many-strain pathogens. Proc. Natl Acad. Sci. USA 99, 17209-17214 (2002).

26. Anderson, R. M. \& May, R. M. Coevolution of hosts and parasites. Parasitology 85, 411-426 (1982).

27. Sasaki, A. \& Dieckmann, U. Oligomorphic dynamics for analyzing the quantitative genetics of adaptive speciation. J. Math. Biol. 63, 601-635 (2011)

28. Kimura, M. A stochastic model concerning the maintenance of genetic variability in quantitative characters. Proc. Natl Acad. Sci. 54, 731-736 (1965).

29. LANDE, R. Maintenance of genetic variability by mutation in a polygenic character with linked loci. Genet. Res. 26, 221-235 (1975).

30. Sasaki, A. Evolution of antigen drift/switching: continuously evading pathogens. J. Theor. Biol. 168, 291-308 (1994).

31. Haraguchi, Y. \& Sasaki, A. Evolutionary pattern of intra-host pathogen antigenic drift: effect of cross-reactivity in immune response. Philos. Trans. $R$. Soc. Lond. B 352, 11-20 (1997).

32. Bull, J. J. \& Ebert, D. Invasion thresholds and the evolution of nonequilibrium virulence. Evol. Appl. 1, 172-182 (2008).

33. Matsuzaki, Y. et al. Neutralizing epitopes and residues mediating the potential antigenic drift of the hemagglutinin-esterase protein of influenza $C$ virus. Viruses 10, 417 (2018).

34. Zambon, M. C. Epidemiology and pathogenesis of influenza. J. Antimicrob. Chemother. 44, 3-9 (1999).

35. Yamashita, M., Krystal, M., Fitch, W. M. \& Palese, P. Influenza B virus evolution: co-circulating lineages and comparison of evolutionary pattern with those of influenza A and C viruses. Virology 163, 112-122 (1988).

36. Medina, R. A. \& García-Sastre, A. Influenza A viruses: new research developments. Nat. Rev. Microbiol. 9, 590-603 (2011).

37. Fedson, D. S. Pandemic influenza and the global vaccine supply. Clin. Infect. Dis. 36, 1552-1561 (2003).

38. Subramanian, R., Graham, A. L., Grenfell, B. T. \& Arinaminpathy, N. Universal or specific? A modeling-based comparison of broad-spectrum influenza vaccines against conventional, strain-matched vaccines. PLoS Comput. Biol. 12, el005204 (2016).

39. Christiansen, F. B. On conditions for evolutionary stability for a continuously varying character. Am. Nat. 138, 37-50 (1991).

40. Geritz, S. A. H., van der Meijden, E. \& Metz, J. A. J. Evolutionary dynamics of seed size and seedling competitive ability. Theor. Popul. Biol. 55, 324-343 (1999).

41. LANDE, R. Quantitative genetic analysis of multivariate evolution, applied to brain:body size allometry. Evolution 33, 402-416 (1979). 
42. LANDE, R. Models of speciation by sexual selection on polygenic traits. Proc. Natl Acad. Sci. USA 78, 3721-3725 (1981).

43. Boots, M., White, A., Best, A. \& Bowers, R. How specificity and epidemiology drive the coevolution of static trait diversity in hosts and parasites. Evolution 68, 1594-1606 (2014).

44. Koelle, K., Cobey, S., Grenfell, B. \& Pascual, M. Epochal evolution shapes the phylodynamics of interpandemic influenza A (H3N2) in humans. Science 314, 1898-1903 (2006)

45. Bedford, T. et al. Global circulation patterns of seasonal influenza viruses vary with antigenic drift. Nature 523, 217-220 (2015).

46. Bedford, T., Rambaut, A. \& Pascual, M. Canalization of the evolutionary trajectory of the human influenza virus. BMC Biol. 10, 38 (2012).

47. Zinder, D., Bedford, T., Gupta, S. \& Pascual, M. The roles of competition and mutation in shaping antigenic and genetic diversity in influenza. PLoS Pathog 9, e1003104 (2013).

48. Eguia, R. et al. A human coronavirus evolves antigenically to escape antibody immunity. PLoS Pathog. 17, e1009453 (2020).

49. Gandon, S., Mackinnon, M. J., Nee, S. \& Read, A. F. Imperfect vaccines and the evolution of pathogen virulence. Nature 414, 751-756 (2001).

50. Read, A. F. et al. Imperfect vaccination can enhance the transmission of highly virulent pathogens. PLoS Biol. 13, e1002198 (2015).

51. Day, T., Gandon, S., Lion, S. \& Otto, S. P. On the evolutionary epidemiology of SARS-CoV-2. Curr. Biol. 30, R849-R857 (2020).

52. Visher, E. et al. The three Ts of pathogen evolution during zoonotic emergence. Preprint at https://ecoevorxiv.org/tueyb/ (2021).

53. Chesson, P. Mechanisms of maintenance of species diversity. Annu. Rev. Ecol. Syst. 31, 343-366 (2000).

\section{Acknowledgements}

This study was supported by grants from the NIH R01 GM122061-03 and the NSF DEB- 2011109 to M.B.; ANR JCJC grant ANR-16-CE35-0012-01 to S.L.; and the ESB Cooperation Program, The Graduate University for Advanced Studies, SOKENDAI to A.S.

\section{Author contributions}

M.B., A.S. and S.L. contributed to the conception of the project, the writing of the original draft, editing and revision of the paper. A.S. and S.L. carried out the analysis of the model.

\section{Competing interests}

The authors declare no competing interests.

\section{Additional information}

Extended data is available for this paper at https://doi.org/10.1038/s41559-021-01603-z.

Supplementary information The online version contains supplementary material available at https://doi.org/10.1038/s41559-021-01603-z.

Correspondence and requests for materials should be addressed to Mike Boots. Peer review information Nature Ecology \& Evolution thanks the anonymous reviewers for their contribution to the peer review of this work.

Reprints and permissions information is available at www.nature.com/reprints. Publisher's note Springer Nature remains neutral with regard to jurisdictional claims in published maps and institutional affiliations.

(C) The Author(s), under exclusive licence to Springer Nature Limited 2021 


\section{(a) Low Cross Immunity}

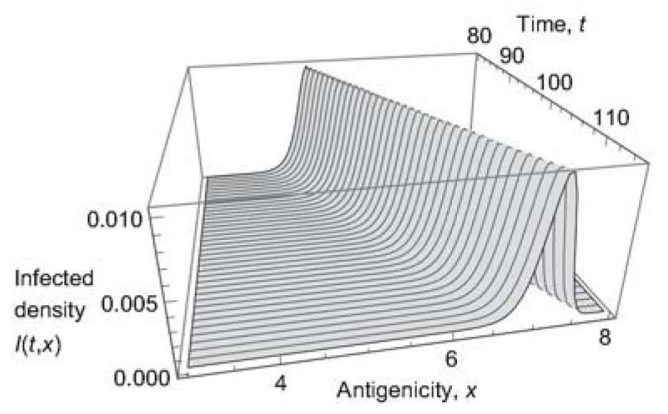

80

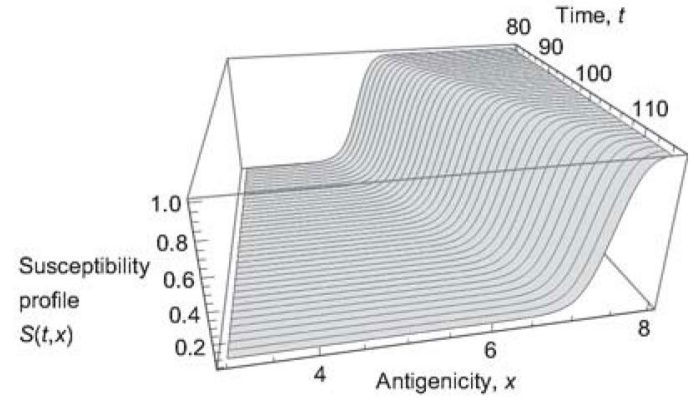

(c)

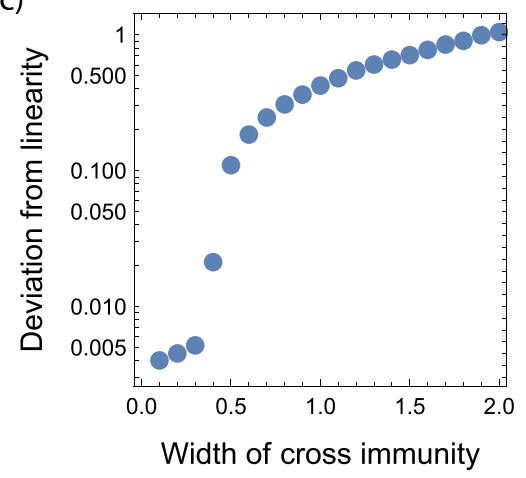

(b) High Cross Immunity
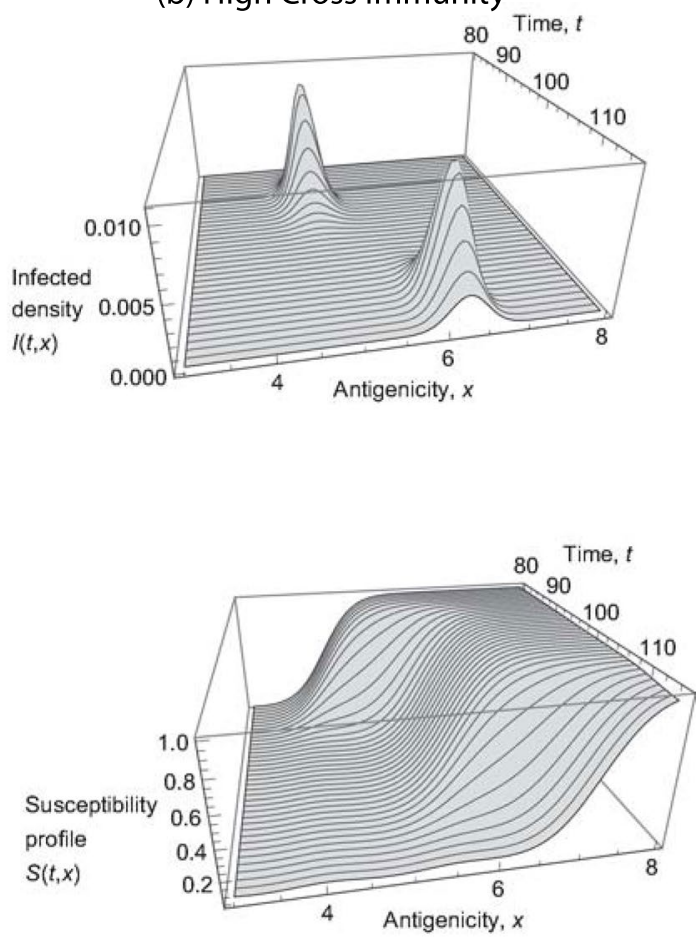

(d)

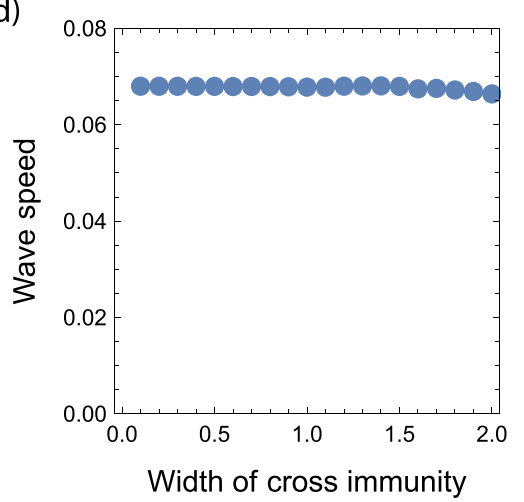

Extended Data Fig. 1 | Continuous antigenic drift (a) and periodical antigenic shifts (b) of the model. The surface in upper panel denotes the infected density $I(t, x)$ varying in time $t$ and antigenicty $x$, and the surface in lower panel denotes the density of hosts $S(t, x)$ that are susceptible to antigenicity variant $x$ of pathogen at time $t$. The width of cross immunity is $\omega=0.2$ in (a) and $\omega=0.6$ in (b), where we assume a Gaussian form for cross immunity function: $\sigma(d)=\exp \left(-d^{2} / 2 \omega^{2}\right)$. A continuous antigenic drift solution (travelling wave of a fixed shaped profile with a constant wave speed) loses stability around $\omega=0.4$ and the departure from the travelling wave increases as $\omega$ increases (c). The wave speeds stay nearly constant and agree with (3) $(v=\sqrt{2(\beta-\alpha-\gamma) D}=0.0748)$ when $\omega$ is varied (d). Other parameters are $\beta=2, \alpha=0.1, \gamma=0.5$ and $D=0.001$. 
(a)

Kullback-Leibler divergence between predicted and true trajectories Actual antigenicity jump occured at $t=54.6061$

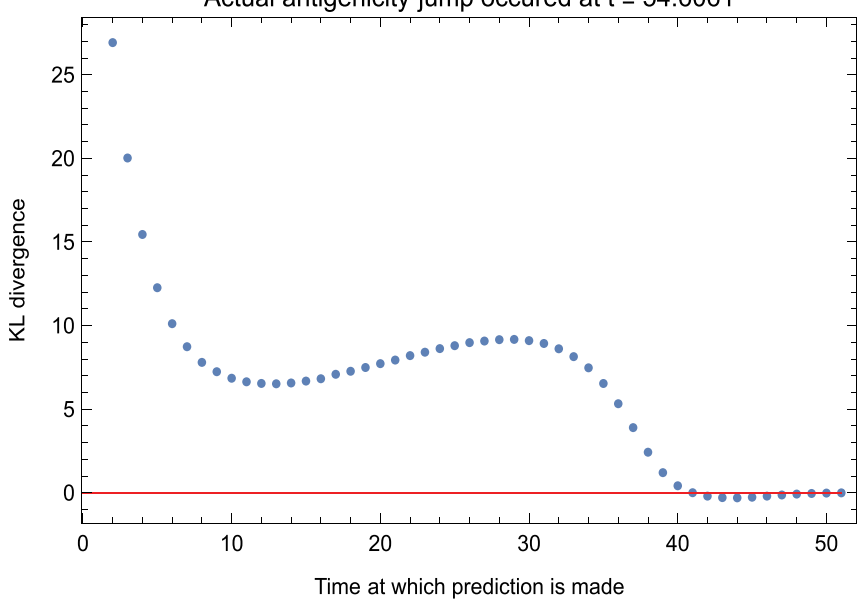

(b)

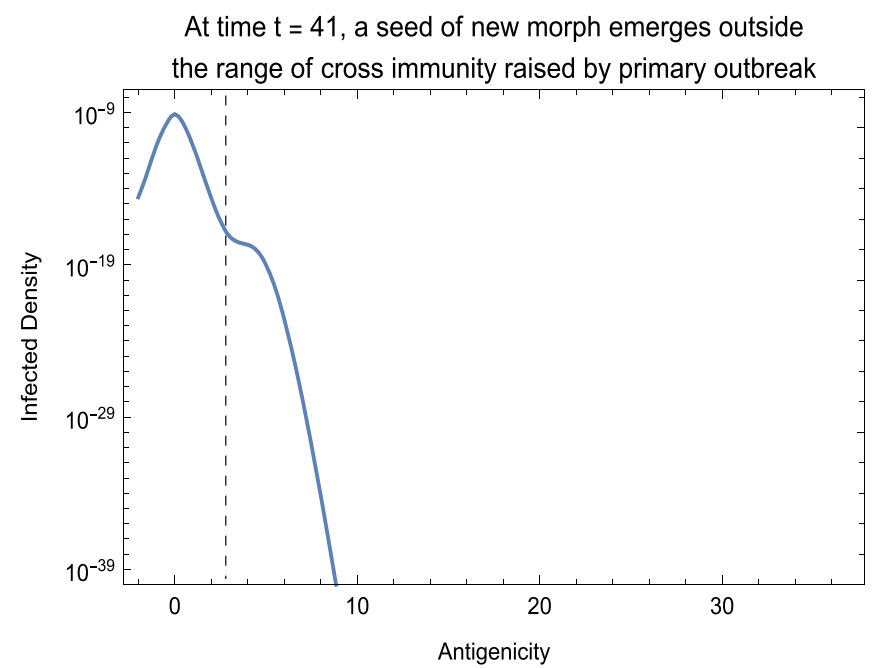

Extended Data Fig. 2 | The accuracy of prediction of the second outbreak as a function of the prediction timing. Using the Kullback-Leibler divergence (left panel) (a) The $K L$ divergence plotted here is $I_{K L}(P, Q)=\frac{1}{t_{1}-t_{0}} \int_{t}^{t_{1}} Q(t) \log Q(t) / P(t)$, where $Q(t)$ is the trajectory for mean antigenicity observed in simulation, and $P(t)$ is the corresponding trajectory obtained with OMD with using the data at $t=t_{0}$ being used to set the initial condition. The right end of the comparison in time horizon is set to $t_{1}=80$ when the second outbreak is over. (b) The logarithmic density of antigenicity variants, $I(t, x)$, at $t=41$. A tiny seed for the second morph around $x=6$ is visible. 


\section{Reporting Summary}

Nature Research wishes to improve the reproducibility of the work that we publish. This form provides structure for consistency and transparency in reporting. For further information on Nature Research policies, see our Editorial Policies and the Editorial Policy Checklist.

\section{Statistics}

For all statistical analyses, confirm that the following items are present in the figure legend, table legend, main text, or Methods section.

n/a Confirmed

Х $\square$ The exact sample size $(n)$ for each experimental group/condition, given as a discrete number and unit of measurement

Х $\square$ A statement on whether measurements were taken from distinct samples or whether the same sample was measured repeatedly

$\triangle$ The statistical test(s) used AND whether they are one- or two-sided

Х $\square$ Only common tests should be described solely by name; describe more complex techniques in the Methods section.

Х $\square$ A description of all covariates tested

Х $\square$ A description of any assumptions or corrections, such as tests of normality and adjustment for multiple comparisons

$\nabla \square$ A full description of the statistical parameters including central tendency (e.g. means) or other basic estimates (e.g. regression coefficient)

X $\square$ AND variation (e.g. standard deviation) or associated estimates of uncertainty (e.g. confidence intervals)

X $\square$ For null hypothesis testing, the test statistic (e.g. $F, t, r$ ) with confidence intervals, effect sizes, degrees of freedom and $P$ value noted

\ive $P$ values as exact values whenever suitable.

Х $\square$ For Bayesian analysis, information on the choice of priors and Markov chain Monte Carlo settings

Х $\square$ For hierarchical and complex designs, identification of the appropriate level for tests and full reporting of outcomes

\ $\square$ Estimates of effect sizes (e.g. Cohen's $d$, Pearson's $r$ ), indicating how they were calculated

Our web collection on statistics for biologists contains articles on many of the points above.

\section{Software and code}

Policy information about availability of computer code

Data collection There is no data collected

Data analysis We do not analyse data

For manuscripts utilizing custom algorithms or software that are central to the research but not yet described in published literature, software must be made available to editors and reviewers. We strongly encourage code deposition in a community repository (e.g. GitHub). See the Nature Research guidelines for submitting code \& software for further information.

\section{Data}

Policy information about availability of data

All manuscripts must include a data availability statement. This statement should provide the following information, where applicable:

- Accession codes, unique identifiers, or web links for publicly available datasets

- A list of figures that have associated raw data

- A description of any restrictions on data availability 


\section{Field-specific reporting}

Please select the one below that is the best fit for your research. If you are not sure, read the appropriate sections before making your selection.

$\square$ Life sciences $\quad \square$ Behavioural \& social sciences $\quad \square$ Ecological, evolutionary \& environmental sciences

For a reference copy of the document with all sections, see nature.com/documents/nr-reporting-summary-flat.pdf

\section{Ecological, evolutionary \& environmental sciences study design}

All studies must disclose on these points even when the disclosure is negative.

Study description
Research sample
Sampling strategy
Data collection
Timing and spatial scale N/A
Data exclusions
Reproducibility
Randomization N/A
Blinding
Did the study involve field work? $\square$ Yes X No

\section{Reporting for specific materials, systems and methods}

We require information from authors about some types of materials, experimental systems and methods used in many studies. Here, indicate whether each material, system or method listed is relevant to your study. If you are not sure if a list item applies to your research, read the appropriate section before selecting a response.

Materials \& experimental systems

$\mathrm{n} / \mathrm{a}$ Involved in the study

Х $\square$ Antibodies

Х $\square$ Eukaryotic cell lines

Х $\square$ Palaeontology and archaeology

$\bigotimes \square$ Animals and other organisms

\ $\square$ Human research participants

\ $\square$ Clinical data

$\bigotimes \square$ Dual use research of concern

\begin{tabular}{l|l} 
Methods \\
\hline n/a & Involved in the study \\
$\searrow$ & $\square$ ChIP-seq \\
$\searrow$ & $\square$ Flow cytometry \\
$\triangle$ & $\square$ MRI-based neuroimaging
\end{tabular}

